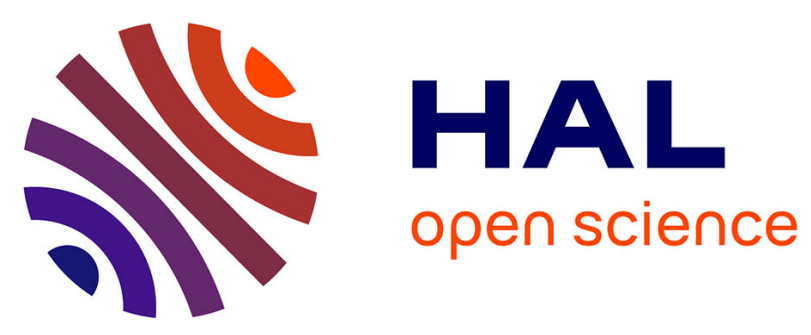

\title{
A survey of challenges and solutions for the integration of renewable energy in datacenters
}

Gustavo Rostirolla, Léo Grange, Minh-Thuyen Thi, Patricia Stolf, Jean-Marc

Pierson, Georges da Costa, Gwilherm Baudic, Marwa Haddad, Ayham

Kassab, Jean-Marc Nicod, et al.

\section{To cite this version:}

Gustavo Rostirolla, Léo Grange, Minh-Thuyen Thi, Patricia Stolf, Jean-Marc Pierson, et al.. A survey of challenges and solutions for the integration of renewable energy in datacenters. Renewable and Sustainable Energy Reviews, 2021, 155, pp.111787 - 111805. 10.1016/j.rser.2021.111787 . hal03549336

\section{HAL Id: hal-03549336 \\ https://hal.science/hal-03549336}

Submitted on 31 Jan 2022

HAL is a multi-disciplinary open access archive for the deposit and dissemination of scientific research documents, whether they are published or not. The documents may come from teaching and research institutions in France or abroad, or from public or private research centers.
L'archive ouverte pluridisciplinaire HAL, est destinée au dépôt et à la diffusion de documents scientifiques de niveau recherche, publiés ou non, émanant des établissements d'enseignement et de recherche français ou étrangers, des laboratoires publics ou privés. 


\section{Highlights}

\section{A survey of challenges and solutions for the integration of renewable energy in datacenters}

Rostirolla, G.,Grange, L.,Minh-Thuyen, T.,Stolf, P.*,Pierson, J.M.,Da Costa, G.,Baudic, G.,Haddad, M.,Kassab, A.,Nicod, J.M.,Philippe, L.,Rehn-Sonigo, V.,Roche, R.,Celik, B.,Caux, S.,Lecuivre, J.

- Challenges and solutions for using of renewable energy in datacenters are surveyed

- Related work on modeling, sizing and managing power and IT resources is presented

- Flexibility on power and IT to tackle generation intermittency is investigated

- Open research challenges regarding infrastructure and predictive methods are listed

Abstract: As more and more activities are moving online, the need for datacenters is increasing which raises their energy consumption and the resulting greenhouse gas emissions. The supply of datacenters with renewable energy is often seen as the main solution to this nexus. However, multiple challenges are posed by their integration. Solutions to these challenges, mainly in the form of flexibility and energy efficiency, require multidisciplinary skills. As a consequence, this article aims to review scientific efforts in several complementary disciplines: electrical engineering, computer science, control system engineering and operations research. It covers datacenter architecture, infrastructure sizing and resource management on both information and energy sides. Conclusions on current progresses are drawn and challenges that remain to be addressed are listed as well.

Keywords: Datacenters Renewable energy Energy scheduling IT management

Word count: 10099

List of abbreviations

- DVFS Dynamic Voltage and Frequency Scaling

- VM Virtual Machine

- IT Information Technology

- ICT Information and Communication Technology

- RES Renewable Energy Sources

- HRES Hybrid Renewable Energy Sources

- SLA Service Level Agreement

- UPS Uninterruptible Power Supply

- NSRDB National Solar Radiation Data Base

- PV Photo-Voltaic

- ISO International Standardization Organization

- IEC International Electrotechnical Commission

- PUE Power Usage Effectiveness 
A survey of challenges and solutions for the integration of renewable energy in datacenters

- REF Renewable Energy Factor

- ERF Energy Reuse Factor

- HSS Hydrogen Storage System

- WT Wind Turbine

- SHP Small Hydropower Systems

- MHP Micro Hydropower Systems

- AH Autonomy Hours

- DOD Depth of Discharge

- SOC State of Charge

- BES Battery Energy Storage

- LHV Lower Heating Value

- HHV Higher Heating Value

- PEMFC Proton Exchange Membrane Fuel Cells

- GA Genetic Algorithm

- ESD Energy Storage Device

- GHG Greenhouse Gas

- PSO Particle Swarm Optimization

- CAPEX Capital Expense

- OPEX Operating Expense

- OM Operation and Maintenance

- LCOE Levelized Cost of Energy

- LACE Levelized Avoided Cost of Electricity

- DC Direct Current

- AC Alternating Current

- CHP Combined Heat and Power

- SC Super-Capacitor

- FC Fuel Cell

- HEB Heterogeneous energy buffering

- LCE Leveled Cost of Electricity

- LPS Loss of Power Supply

- EASC Energy Adaptive Software Controller

- GLB Graphical Load Balancing

- EON Elastic Optical Network

- CaaS Container as-a-Service

- HPC High Performance Computing

- TCO Total Cost of Ownership

- LCA Life Cycle Analysis

- GDN GreenDataNet

- CPPA Corporate Purchase Agreement

- PDU Power Distribution Unit

- HVAC Heating, Ventilation and Air Conditioning 


\title{
A survey of challenges and solutions for the integration of renewable energy in datacenters ${ }^{\star}, \star \star$
}

\author{
Rostirolla, G. ${ }^{a, d}$, Grange, L. ${ }^{a}$, Minh-Thuyen, T. ${ }^{a}$, Stolf, P.*a ${ }^{*}$ Pierson, J.M. ${ }^{a}$, Da Costa, G. ${ }^{a}$, \\ Baudic, G. ${ }^{a}$, Haddad, M. ${ }^{b}$, Kassab, A. ${ }^{b}$, Nicod, J.M. ${ }^{b}$, Philippe, L. ${ }^{b}$, Rehn-Sonigo, V. ${ }^{b}$, \\ Roche, R. ${ }^{c}$, Celik, B. ${ }^{d}$, Caux, S. $^{d}$ and Lecuivre, J. ${ }^{e}$ \\ ${ }^{a}$ Université de Toulouse, Institut de Recherche en Informatique de Toulouse (IRIT), Toulouse, France \\ ${ }^{b}$ FEMTO-ST Institute, CNRS, Univ. Bourgogne Franche-Comté, UFC/ENSMM, Besançon, France \\ ${ }^{c}$ FEMTO-ST Institute, CNRS, Univ. Bourgogne Franche-Comté, UTBM, Belfort, France \\ ${ }^{d}$ Université de Toulouse, Laplace UMR5213, Toulouse, France \\ ${ }^{e}$ EATON SAS, Grenoble, France
}

\section{ARTICLE INFO}

\section{Keywords:}

Datacenters

Renewable energy

Energy scheduling

IT management

\begin{abstract}
A B S T R ACT
As more and more activities are moving online, the need for datacenters is increasing which raises their energy consumption and the resulting greenhouse gas emissions. The supply of datacenters with renewable energy is often seen as the main solution to this nexus. However, multiple challenges are posed by their integration. Solutions to these challenges, mainly in the form of flexibility and energy efficiency, require multidisciplinary skills. As a consequence, this article aims to review scientific efforts in several complementary disciplines: electrical engineering, computer science, control system engineering and operations research. It covers datacenter architecture, infrastructure sizing and resource management on both information and energy sides. Conclusions on current progresses are drawn and challenges that remain to be addressed are listed as well.
\end{abstract}

\section{Introduction}

The ever growing use of online services or applications run from terminals (mobile phones, laptops, desktop computers), leads to increasing needs for computing facilities, and to the building of new datacenters and the expansion of existing ones. These datacenters generate carbon emissions due to their consumption of electric energy, which is mostly generated from fossil fuels such as coal or natural gas. However, with the awareness of their ecological impact, the minimization of their environmental footprint linked to the reduction of their energy consumption is becoming a major concern. According to the International Energy Agency, in 2019, while the energy efficiency of datacenters and data networks has improved significantly over the past few years, the electricity consumption of datacenters is estimated at $200 \mathrm{TWh}$ or about $1 \%$ of the world electricity consumption, with similar numbers for data networks $[1,2,3]$. Moreover, partly due to the growing use of mobile devices and the development of technologies like 5G, datacenter energy consumption is expected to increase by $9 \%$ per year in the near future.

In a context of growing concerns over climate change and its consequences, the Information and Communication Technology (ICT) industry, through the ITU standard ITU-T L.1470 [4] agreed in 2020 on a target to reduce emissions by $45 \%$ between 2020 and 2030 to help reach the global $1.5^{\circ} \mathrm{C}$ trajectory.

To make this goal reachable, two complementary approaches can be followed: improving the energy efficiency of Information Technology (IT) components, and changing the way the electric energy is produced and supplied to the datacenter. Several initiatives address the problem of energy efficiency, which has been covered by a large number of studies in the literature. Initiatives may focus on processor consumption control, using Dynamic Voltage and Frequency Scaling (DVFS) for instance, or on consolidation, i.e., clustering virtual machines to avoid powering on too many physical hosts. For example, in [5], the authors present a survey on techniques for improving the energy efficiency of large scale distributed systems. In [6], the authors present a taxonomy of power and energy management dividing the

\footnotetext{
${ }^{\star}$ This document is the results of the DataZero research project funded by the ANR (ANR-15-CE25-0012).

${ }^{\star \star}$ The authors are arranged institution by institution. Electrical part by FEMTO-ST Institute, IT part by Université de Toulouse.

*Corresponding author: patricia.stolf@irit.fr

ORCID(s):
} 
techniques into static and dynamic ones and detailing them at different levels, e.g., virtualization, operating system, and firmware/hardware level. In [7], the authors focus on the energy efficiency of ICT equipment in cloud computing with a separate focus for servers and network. Finally a more recent survey by [8] provides a categorization for the problem of energy efficiency in computing, and presents various methodologies for green datacenters at host level, network level, thermal-aware techniques, virtualized level and geographic level. Some approaches also focus on electric distribution efficiency, and consider Direct Current (DC) supply instead of Alternating Current (AC) supply [9]. The reduction of energy consumption however tends to have a limited impact, since it often decreases IT performance (i.e., applications may finish later or may be delayed). It may even lead to rebound effects as users tend to use more computing resources if they are cheaper [3]. DC supply is also subject to several challenges, for example related to circuit breaking. Finally, other efforts on the recovery of waste heat generated by datacenters have been published [10,11]. Energy efficiency is therefore necessary but only represents one part of the solutions needed to satisfy the ever growing need for computing power while reducing the carbon emissions.

The second approach to limit the carbon footprint relies on using cleaner energy sources, i.e., sources without direct carbon emission. [12] reviews the use of renewable energies in the BRICS countries and details financial models: investment, government policies... [13] presents carbon reduction and its financial market. In datacenters context, several IT companies, often under public pressure, have announced to use more green energy in their datacenters and several initiatives have been launched. Apple [14], for example, will supply $200 \mathrm{MW}$ of solar power for their datacenter in Reno, Nevada. Google [15] has launched two projects in Alabama and Tennessee for the building of farms that will produce $300 \mathrm{MW}$ of solar energy. In parallel, many researchers are working on the use of renewable energy sources and storage units to supply datacenters. The "GreenDataNet" European project [16] has proposed an optimization solution, where solar panels and batteries are combined to supply urban datacenters. At the same time, academic efforts have been made to improve energy efficiency using renewable energy in datacenters. Design strategies have been proposed to make datacenters less dependent on grid energy and more energy efficient at both the infrastructure level (IT equipment, cooling systems and network topology [17]) and IT workload management level [18, 19]. Similarly, the "RenewIT" project [20] has developed a simulation tool for datacenter operators to select the optimum combination of efficiency measures and renewables for energy and carbon sustainable facilities in several European climate regions.

However, the integration of renewable energy in datacenters causes technical difficulties. Datacenters can either generate their own renewable energy (self-generation), draw it from a nearby plant (co-location) or simply buy renewable energy from an external supplier on markets or via a power purchase agreement. In the case where a datacenter would be supplied using only local renewable energy, real-time balancing of supply and demand is a challenge that must take into account (i) the needs of datacenters for highly reliable power supply to support their quality of service, (ii) the fluctuations of their load that may be large between the peak and the usual case, and (iii) the variability, intermittency and non-dispatchable properties of renewable power generation. As a consequence, supplying a datacenter using only intermittent renewables is currently unfeasible, at reasonable cost and reliability, without adding energy storage or conventional generators consuming fossil fuels, which adds to the complexity and costs of designing a datacenter and its attached infrastructure.

Several surveys, such as [21], [22] and [5], have already presented the issues and works linked to the energy consumption of IT equipment in the literature. In [23], Kong et al. present a survey on green-energy-aware power management for datacenters. They focus on works tackling the scheduling of workloads and Virtual Machine (VM) management in datacenters supplied by green energy and only provide a short description of a few works considering energy capacity planning.

In this survey, we propose a multidisciplinary review of the scientific literature on challenges and solutions to enable the development of green and energy efficient datacenters. The paper contributions are as follows: i) we consider studies from the fields of electrical engineering, control systems and computer science, as only considering one discipline would only provide a partial view of all the possible challenges and approaches; ii) we focus on the coupling of datacenters with intermittent renewable generation, with flexibility sources coming from both power supply and IT consumption; iii) we cover not only the operation of the datacenter but also its design phases, on both power supply and IT aspects. Finally, we consider new contributions published since 2014, when [23] was published.

The remainder of this article is organized as follows. In Section 2 we synthesize works focusing on the energy infrastructure of datacenters with renewable energy. The management of energy sources and works studying IT management, scheduling, and singular vs. distributed datacenters to increase the use of renewable energy in datacenters are then presented in Section 3. Finally, in Section 4, the paper is concluded and some open research challenges of the domain are presented. 


\section{Datacenter Energy Infrastructure}

In this section, we present the works focusing on the design and assessment of the energy infrastructure of datacenters powered by renewable energy sources.

\subsection{On/Off Site Generation}

Two approaches are usually followed when trying to reduce the use of brown (fossil-fuel based) energy in datacenters, besides compute/load management: using local renewable resources (on-site generation) and/or resources available on the grid (off-site generation) [24]. The preferred approach is chosen by IT companies depending on various factors such as financial interests, grid reliability, and resource availability and geographical location. A major issue is the intermittent and uncertain character of Renewable Energy Sources (RES), which can be dealt with by using additional resources and/or curtailment. However, IT companies, rather than to deal with the issue themselves, might prefer to use already existing RES on the electricity grid. This option can influence local generation companies and/or the regional utility to build more renewable resources to attract IT companies through long-term agreements. For example, MidAmerican Energy, a utility in Iowa, decided to invest $\$ 1.9$ billion to increase its wind generation after a renewable energy policy announcement of Facebook [25]. Similarly, Google [26] operates its datacenters using 100\% renewable energy since 2017, but this energy is not generated on-site. With this approach, there is no guarantee that the electrons supplying the datacenter are actually "green", as, due to the laws of physics, they will typically originate from the nearest plants, green or not.

On the other hand, if the current electricity grid is not able to support a high penetration of RES, if the datacenter operator requires the use of clean energy or if the operator requires greater control on energy supply, companies might be interested in investing in on-site generation, either from conventional or from renewable generators. IT companies then avoid the incurred transmission and distribution costs, as well as reduce the system losses [27]. Large companies such as Apple, Google or eBay are for example building unplugged datacenters to have a better control over their infrastructure. eBay's Utah datacenter uses natural gas to produce its electricity [28], while the grid is only used as a backup. In a similar fashion, Apple's Maiden, North Carolina datacenter [29], is using a $40 \mathrm{MW}$ solar plant along with biogas fuel cells. Similarly, Apple has invested in several on-site installations of renewable energy, in particular solar power in North Carolina and Nevada [30]. A drawback is that this type of installation requires large financial investment, which might not be possible for some small IT companies. Additionally, such energy infrastructure is considerably more complex to operate if it is not grid-connected, as a balance between generation and load must be ensured in real-time [31, 32, 33, 34]. Fig. 1 shows a comparison of the load of a small datacenter with the solar generation measured in the same location over a period of 5 days. It can be observed that both profiles do not match (even if their average value is the same), which implies the need for additional devices such as storage as well as load management strategies further discussed in the next sections. As a consequence, reliability, stability and costs are impacted.

\subsection{Renewable Energy Sources}

RES are widely seen as one of the main solutions to help decarbonize electricity production, which includes datacenters. While renewable energy is already integrated in today's power systems to a certain extent, reaching large shares (if not 100\% [35]) of intermittent and variable renewable generation poses multiple technical and economical challenges [36]. Multiple types of RES are commonly used with datacenters or are expected to grow significantly in the future. In the following, we discuss the interest of the different types of RES from the point-of-view of a datacenter application.

\subsubsection{Wind turbines}

Wind turbine generator output is approximately proportional to the cube of the wind speed measured at the turbine hub, rated power ranges from a few $\mathrm{kW}$ for the smallest to multi-MW values for the largest. For most datacenter applications, where transient behavior is not particularly studied, simple models can be used, such as the ones proposed by Garcia et al. [37] and other researchers [38, 39, 40, 41]. A challenge related to wind energy is that wind resources are location-dependent (and often not close to the consumption location) and sometimes very variable, in addition to a low energy density in terms of area used. Wind farm operation is carbon-free, with limited noise. Acceptability from the general public is however variable, as some consider turbines as visual pollution. As a consequence, to the authors' best knowledge, no major datacenter runs entirely on local wind power. More and more datacenter operators, such as Amazon [42] with 91 MW planned in Sweden or Apple [43] with 200 MW in Oregon, resort to Corporate 


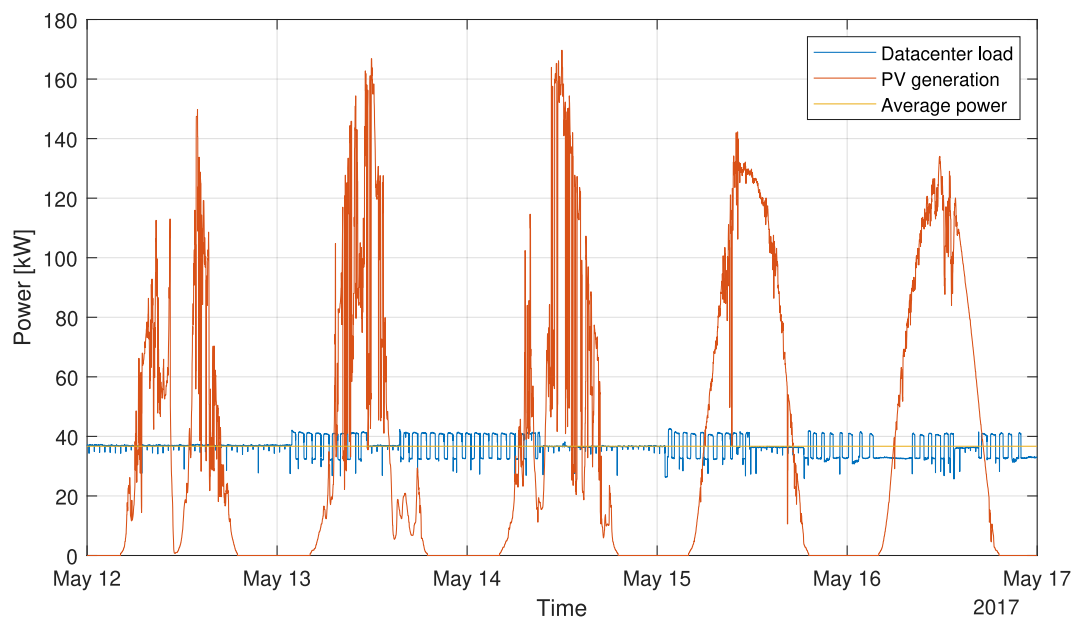

Figure 1: Comparison made by the authors of the load of a small datacenter (including cooling) and the generation from a fictitious photovoltaic plant in Belfort, France, over a period of 5 days. Both power profiles have the same average value.

Purchase Agreement (CPPA) with grid-connected wind farms to secure wind energy supply at a fixed price, but remain connected to the grid. In a different perspective, project WindCores [44] in Germany aims to install small datacenters inside wind turbines, enabling the datacenter to be powered at $92 \%$ from local wind power.

\subsection{Photovoltaics}

Photo-Voltaic (PV) panels convert light into electricity using the photovoltaic effect. Simple models assume that PV output is proportional to the solar radiation measured on the panels, although more elaborate models can also consider other effects, such as those related to temperature and variable efficiency levels [45, 46, 47, 48, 49, 41]. This type of energy suffers from challenges similar to those of wind energy, especially in terms of intermittency and density. Several differences exist, including low efficiency, absence of noise, limited visual pollution, the presence of solar radiation throughout the world (although sometimes with low intensity), and the fact that daily patterns of generation can be observed due to day/night cycles. As for wind power, PV power is often used to supply datacenters through CPPA, either from large, utility-size plants (100 MW for Facebook in New Mexico [50], $413 \mathrm{MW}$ for Google in Alabama and Tennessee [51]) or from a large number of rooftop PV systems (50 MW for Facebook [52] in Singapore).

\subsubsection{Hydropower}

Hydroelectric power is currently the world's main RES and consists in the conversion of water flow into electric power through turbines. Rated power ranges from $\mathrm{kW}$ to GW. Regarding modeling, according to Kishor et al. [53], models of hydro plants can be classified into both linear models (non-elastic) and nonlinear models (elastic) depending on the complexity of the involved equations. In the simplest models, the power output is proportional to the flow of water and the waterfall head. While hydropower is a mature, cheap and mostly carbon-free RES, it is heavily locationdependent (i.e., there must be a river and/or a dam nearby) and can have a significant impact on natural ecosystems, especially due to dams. Compared to wind and solar, its main interest is that the output can be controlled by adjusting the flow of water. Several examples of datacenters running mostly with hydropower exist. A datacenter in the Swiss Alps [54] claims to run with $99.9 \%$ hydropower, through a plant installed on site, and $0.1 \%$ solar power. Other examples can be found in regions with significant hydropower resources such as Sweden (Facebook [55]), Oregon (Apple [56], with small dams), Norway (Volkswagen [57]) or Iceland [58]. Such locations are also often in cold regions, which facilitates infrastructure cooling.

\subsubsection{Biomass}

This technology corresponds to the use of organic material coming from either animals or plants in order to generate heat or electricity. Common examples include wood, crops, manure or human waste. This material can either be burnt directly or converted to biofuels or biogas which can in turn be stored, transported or burnt [59]. Several methods exist to extract useful byproducts from biomass such as: direct combustion [60], pyrolysis [61], gasification [62], 
liquefaction [63], supercritic fluid extraction [64], anaerobic digestion [65], fermentation [66], acid hydrolysis, enzyme hydrolysis, and esterification. Due to the large number of different processes that can be used to convert biomass into electric power, generalizing the advantages and drawbacks of these types of sources is difficult, and as a consequence, so is the identification of the main types of models. Nevertheless, a major challenge of this type of energy is the reliable availability of the biomass resource, due to geographical constraints or due to production variability throughout the year. Several projects based on biomass to supply datacenters have been considered. For example, HP Labs designed a system capable of powering a $1 \mathrm{MW}$ power datacenter using manure of 10,000 cows from a farm [67]. This approach could also provide additional revenue for farmers. Several other biomass projects were considered in 2012 [68], e.g., in Georgia and in Ohio, however it seems that most of them were abandoned.

\subsubsection{Discussion}

From the above, we can notice that all the main types of RES are employed for powering datacenters, either directly or through CPPA. A distinction must however be done between dispatchable (i.e., controllable) sources such as hydro or biomass and non-dispatchable and intermittent sources, such as wind and solar. In the former case, this characteristic is a major difficulty in the perspective of running a datacenter solely with these types of local energy. They however benefit from the facts that they can be installed in many locations, that their emissions are low even over their life cycle [69], and that their life cycle costs have dramatically decreased over time to values below those of fossil fuelbased generation, even without subsidies [70]. In order to further their integration with datacenters and compensate the variability and intermittency of solar and wind, additional equipment or strategies are therefore necessary, such as energy storage as discussed in the next section, demand management [71] or combining different types of RES.

\subsection{Energy Storage Systems}

To enable better RES integration, energy storage systems are required to enable shifting generation and/or consumption over time. They enable storing excess generation for later use, e.g., storing excess PV generation during the day for use at night $[72,73,74,75,76]$. Other applications include the ability to provide ancillary services to the grid [77] [78] (e.g., by increasing or decreasing the datacenter load depending on an external signal provided by the transmission system operator), demand response (e.g., by discharging a battery to reduce the net load on the utility grid in case of congestion or limited generation capacity), or arbitrage (e.g., using local storage to be able to use stored energy or the grid depending on prices) $[79,80,81,82]$. In addition to the above, powering a datacenter solely with RES at all times (i.e., hour by hour and not only on average) requires the use of massive storage capacity. For example, Google plans to use energy from a $350 \mathrm{MW}$ solar plant in Nevada, coupled with a $280 \mathrm{MW}$ battery [83].

There are multiple energy storage technologies, each with its own advantages and drawbacks [81, 84]. Pumped hydropower and underground compressed air storage are by nature limited by geographical, geological and terrain considerations, which makes them unsuitable for most datacenter applications. Short-term storage technologies such as flywheels or supercapacitors are mostly used for their ability to output or absorb large amounts of power over very short durations (ms to minutes), which is essential for stability (not discussed in this paper) for example, but they are not suitable for storing energy even at a horizon of an hour. The two main remaining technologies are batteries and hydrogen, further discussed in the following.

\subsubsection{Battery storage systems}

Batteries are electrochemical devices used to store energy in chemical form. Different types, capacities and battery voltages are available and can be compared by cost, density and behavior [85]. Battery technologies however tend to evolve from lead-acid batteries to lithium-ion batteries or other variants [86] [85]. Despite their higher costs, these new technologies often benefit from better compactness and weight, lower maintenance requirements and longer lifetime. Battery storage systems are mostly utilized in short-term storage scenarios, e.g., over horizons of several hours, such as for day/night cycles. They are however inadequate for storage over longer periods of time due to their non-negligible self-discharge rate and low energy density [87, 88]. Batteries have been used in datacenters as part of Uninterruptible Power Supply (UPS) installations for decades, and are therefore well known. An issue with such systems is however their ageing and capacity and performance degradation over time, which requires them to be replaced when they reach their end-of-life $[89,90]$.

Several modeling approaches have been proposed. The energy-based approach considers batteries as a device in which energy (power) can be added or subtracted, with constraints on state-of-charge and sometimes consideration of self-discharge or ageing [38, 91]. A variant is based on State of Charge (SOC), e.g., a relative level of energy, instead of 
energy $[92,47,76]$. Current-based models are similar but consider currents instead of power and enable determining the battery voltage $[93,49,94,40]$.

\subsubsection{Hydrogen storage systems}

A Hydrogen Storage System (HSS) is composed of an electrolyzer to produce hydrogen from electricity, hydrogen tanks to store this hydrogen, and a fuel cell to produce electric power from hydrogen. Such systems are mostly suitable for long-term storage, e.g., over seasons [95], for several reasons. First, power and energy are decoupled as the power rating depends on the ratings of the fuel cell and the electrolyzer, while the energy stored depends on the size of the tanks. Second, because hydrogen can be compressed, large amounts of energy can be stored over long durations with very low self-discharge (leaks), e.g., to be able to use excess PV generation from summer during winter. Issues related to this type of technology are related to limited dynamics which require hybridization with another form of storage such as batteries, ageing and performance degradation concerns, high costs, low efficiency compared to batteries, and complex safety measures required for pressurized installations. Such installations have also be been proposed for backup power up to 48 hours by Microsoft [96].

In terms of modeling, as for batteries, several levels of detail can be used depending on the intended application. The simplest models can only consider the rated power limits of the fuel cell and the electrolyzer, and the energy capacity of the hydrogen tanks. More detailed models can also consider the voltage-current characteristics of these devices as well as their ageing and limited dynamics [97, 98, 99].

\subsubsection{Discussion}

From the above, we can observe that while energy storage has long been part of datacenters, the range of applications where it is used is expanding, from providing backup supply to a wide variety of objectives related to renewable energy dynamics, markets or grid constraints. While energy storage may not be necessary for a grid-connected datacenter (other than for a UPS), these new use cases increase the motivation for installing such systems. On the other hand, storage is absolutely necessary for a datacenter powered only by local renewable energy. An important challenge related to storage remains costs. While cost estimates have significantly decreased over time [100], they are still considered as prohibitive for many applications [101].

\subsection{Green Datacenter Architecture}

Using local renewable energy in a datacenter implies to change the datacenter energy infrastructure, especially due to constraints resulting from renewable generation intermittency and variability combined with high reliability expectations. In the following we present works on the design of such new infrastructures.

For both industry operators and end-users, operational availability requirements are very high. For example, a tier IV datacenter is expected to reach $99.995 \%$ availability, or 26.3 minutes of annual downtime [102]. Redundancy built in the electric supply of the datacenter is essential for ensuring that such high numbers are reachable, but costs are also necessarily impacted due to over-sizing [103]. As stated in [104], redundancy is necessary to achieve high availability, but it also improves the maintainability, flexibility, expandability and fault management of datacenters. For example, turning off an UPS or a rack in case of maintenance or a fault, servers can remain powered using redundant feeds. Moreover, in case of insufficient reliability, penalties are applied due to a breach of the Service Level Agreement (SLA) with the client of the datacenter. Although this type of agreement highly depends on the application of the tasks running on the servers, this means that a trade-off between availability and costs has to be found, even at the design stage.

Various options are available to enable redundancy. In the literature, [105, 102] describe the most common electrical architectures, which are typically supplied by transmission or distribution grids, depending on their rated power consumption. Architectures range from simple AC feeds from a single upstream transformer to more robust AC supply systems with two separate feeds from different substations. Variants also include DC distribution [9], or mixtures of AC and DC. Batteries are often used in UPS in the case of short interruptions until backup diesel generators start up and take over the UPS. Depending on the expected level of availability, several configurations are used, conventionally from $N$ to $2(N+1)$, where $N$ represents the capacity required to meet the maximum power requested by a datacenter (e.g., $1 \mathrm{MW}$ ). A $N$ datacenter would use two $500 \mathrm{~kW}$ UPS and a single utility feed, while a $N+l$ datacenter would have three $500 \mathrm{~kW}$ UPS, and one of these could be down for maintenance without impact on the operation of the datacenter. $2 N$ means two separate feeds from two different substations and two UPS of $1 \mathrm{MW}$. For $2(N+1)$, the architecture found for $N+1$ datacenters is doubled. More complex architectures, e.g., $3 N / 2$ also exist, but require challenging load 
management systems.

In the presence of on-site renewable generation, these architectures need to be adapted. For example, in [106], RES are integrated in $N+1$ and $2 N$ architectures. Figure 2 presents the $2 N$ architecture with two identical feeds of renewable energy; the $N+1$ architecture consists in adding a fail-safe second electrical source (usually a fuel-based generator or a hydrogen-based one) in parallel to the main electrical source. This structure builds on a new generation UPS known as the GreenDataNet (GDN) UPS [16], used to inject green power in the local infrastructure as shown in Figure 2). This UPS includes photovoltaic panels, wind turbines, fuel cells as well as lithium-ion batteries.

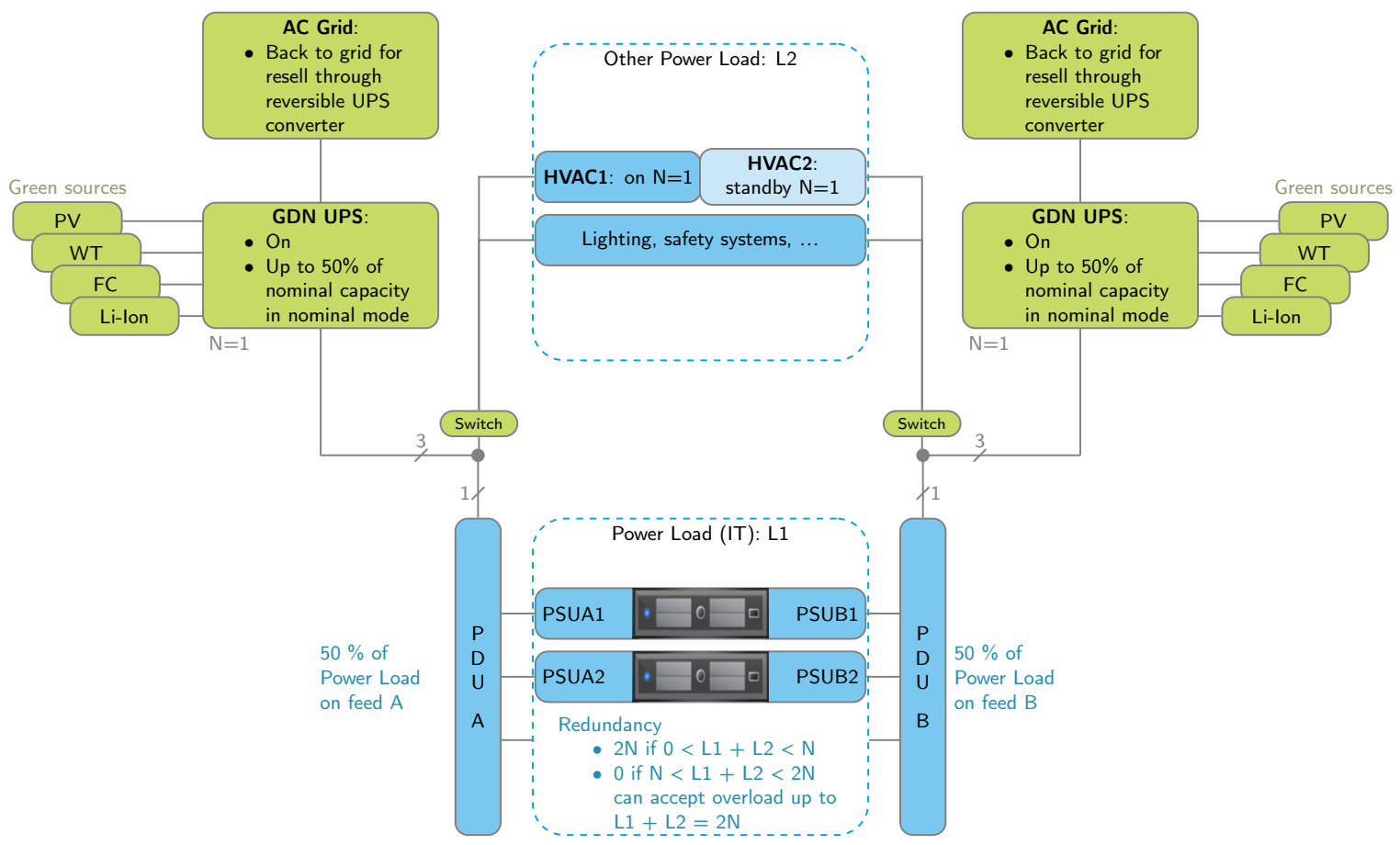

Figure 2: Example of $2 N$ architecture with renewable energy supply: The critical load can be fully supported by each of the RES (photovoltaic panels, wind turbines, fuel cells and lithium-ion batteries) on the right and on the left. Even in case of failure of half of the sources, the datacenter can still operate using $100 \%$ green energy. In addition to servers supplied by a Power Distribution Unit (PDU), Heating, Ventilation and Air Conditioning (HVAC) and other essential loads (e.g., safety systems) also require redundancy, while others (e.g., offices) are considered as less important and may be shed in case of insufficient generation.

\subsection{Sizing of RES and storage}

In the following, we use the term sizing to refer to the process of determining the rated capacity or power of the different RES and storage units. The objective is to predict the size of the primary renewable energy sources and secondary sources (storage devices) in the electrical infrastructure so that these sources are sufficient to ensure the powering of the datacenter on the whole year. In this context, Haddad et al [107] have proposed a global polynomial time sizing process of a standalone datacenter that integrates not only the energy of RES and storage sizing, but also the IT part of the datacenter. Considering a variable energy envelop, different scheduling problems can be proposed with either a polynomial time or a NP-complete complexity [108] having a direct consequence on sizing process complexity. It should allow the infrastructure to produce and store enough energy during periods when there is primary energy and to supply the datacenter even during periods when there is none. While this problem is not new and has long been studied in the literature for microgrids and similar systems [109, 110], mostly for coupling RES with batteries [111], hydrogen [112] and other means of storage. However, few works so far have focused on datacenter applications, especially when considering the specific characteristics of IT load and availability requirements. Several methodologies have been proposed in order to obtain an appropriate composition of energy systems. To facilitate the understanding of these methodologies, we propose in Figure 3 a classification according to the type of methodology used in the reviewed 
literature [110].

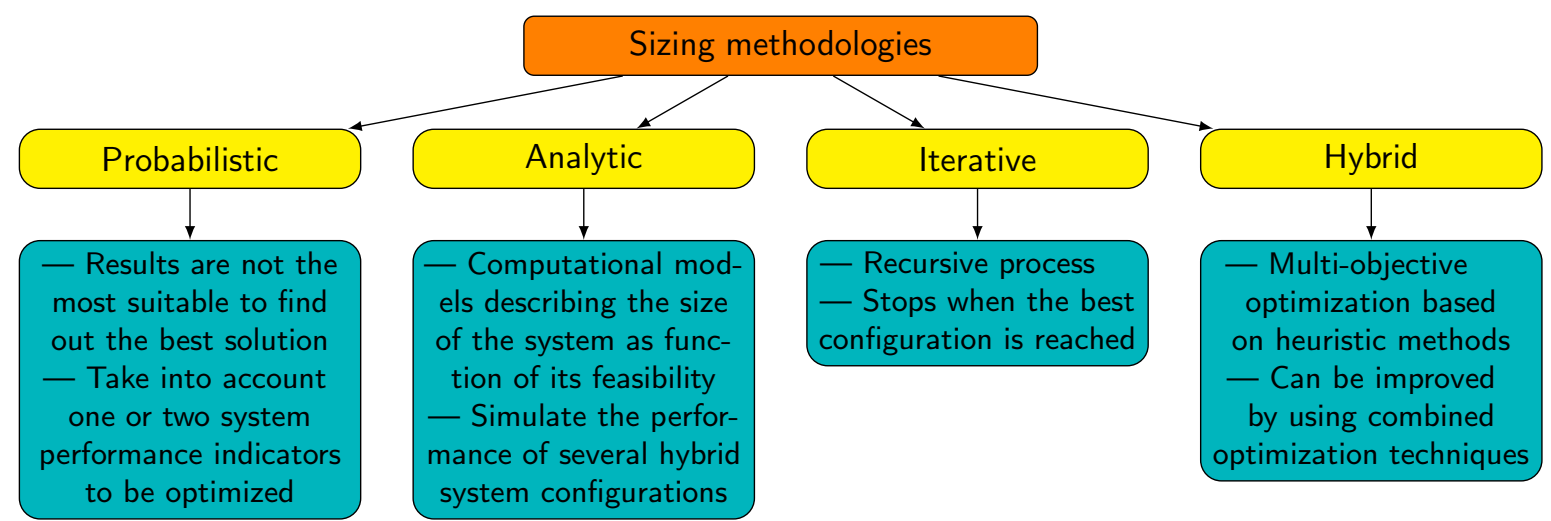

Figure 3: Sizing methodologies.

\subsubsection{Probabilistic methods}

A probabilistic method is a technique based on probability that aims at finding a solution even if the initial problem is combinatory. This method consists in generating a sample space associated with probability. Then given these probabilities it is possible to exhibit solutions from this space that have a probability greater than zero. This method appears as the simplest one to find the appropriate sizing of RES infrastructure. Nevertheless, the method is not efficient to find the best solution because it takes only few optimization criteria into account.

Yang et al. [40] propose a probabilistic method, where they prove the importance of choosing a suitable meteorological year to get an accurate assessment of performance in a hybrid PV-wind energy system. A second probabilistic approach is explained in [41]. The authors propose an approach based on convolution techniques with probability density functions to assess the long term performance of hybrid solar wind power systems. Finally, C. Protogeropoulos et al. introduce two sizing methods [113] for autonomous hybrid systems composed by photo-voltaic panels and wind turbines. The two methods determine the system performance by means of battery to load ratio and energy to load ratio. In the first method the authors use the annual average of the system monthly performance. In the second one, the worst monthly scenario is utilized.

\subsubsection{Analytical methods}

The performance evaluation of a hybrid system in this case can be achieved by computational models (i.e., commercial software tools and/or numerical approximations of system components) describing the RES size in function of its feasibility. Therefore, system performance can be evaluated for a conceivable system architecture set and/or an appropriate component size. The best results are obtained due to the analyze of one or several performance criteria.

The performance evaluation of a hybrid system in this case can be achieved by computational models. Nowadays, several tools have been developed to help the users analyze the integration of the sources for optimizing, designing and evaluating the performance of hybrid systems composed by photo-voltaic panels and wind turbines. This is also presented in detail in a review by Sinha and Chandel [114]. The most used ones are: Hybrid Optimization Model for Electric Renewable Energy (HOMER) [115] Laboratory developed by the National Renewable Energy Laboratory (NREL), the Hybrid Power System Simulation Model (HYBRID2), Hybrid Optimization using Genetic Algorithm etc. However, these pieces of software have some limitations. The most concerning refer to unavailability of some of the software over different platforms, black box design of the components, and the lack of flexibility when compared to other approaches from the literature [114].

\subsubsection{Iterative methods}

Iterative methods in RES sizing consist in using iterative process taking design specifications into account that stop once the convergence criteria is reached, e.g., a given number of iterations or if the best found solution does not improve the objective function for a given number of interactions.

A lot of researchers use Genetic Algorithm (GA) to achieve an optimal sizing. For instance, Kaldellis et al. [48] 
propose to minimize the cost of the system under some constraints. The sizing which represents the optimal solution is reached then by a genetic algorithm process. Similar works can be found in Dufo-Lopez and Bernal-Agustin [116] and Yang et al. [117, 118].

Moreover, Ashok [119] obtained a hybrid system, considered optimal, between several variations for rural communities, decreasing the overall life cycle cost and guaranteeing system reliability. To make it, the authors utilize a numerical algorithm based on a method called QuasiNewton to solve the optimization problem [120].

Another iterative method is reported in [121]. The authors analyze traces from a datacenter of medium size to calculate the optimal size of the solar panels needed to generate enough green energy to cover the entire workload energy consumption, assuming that all the remaining energy produced by the solar panels is stored in a battery without capacity limitation. Then, assuming that the size of the used solar panel is the found ideal size, they calculate the optimal capacity of the battery needed so that the workload consumes zero brown energy. They show that using an opportunistic scheduling algorithm demands a smaller battery size than a baseline algorithm with Energy Storage Device (ESD). Finally the authors combine both approaches in order to find the optimal solution for solar panels and different batteries of different sizes.

\subsubsection{Hybrid methods}

Hybrid methods in RES consist in defining the optimization problem with a multidimensional nature. They aim at solving the sizing problem by a multi-objective optimization based on combining several methods such as heuristic methods but not only (e.g., GA, AI, etc.) and other optimization techniques.

Several researchers $[122,123,124]$ modified GA in order to give the designer the choice of the optimal configuration. This is done by a set of non-dominated Pareto set solutions using selection criteria. In [123], the optimization objectives are the following: (i) minimizing the cost of energy in the system; and (ii) minimize the Greenhouse Gas (GHG) emissions. In this work, the main originality comes from the assessment of GHG emissions based on life cycle analysis. A similar work is proposed in [125] where the authors utilize a particle swarm optimization (PSO) algorithm to obtain a set of Pareto solutions (non dominant). The optimization objective in this work is first techno-economical and then technical, economical and environmental.

Moreover, Artificial Neural Networks can be utilized as a hybrid method for sizing. Some authors [126, 127] utilize the same kind of neural network to predict daily solar radiation in isolated areas as input data for the sizing process when data is missing. It is also used to improve the analytic method of loss of load probability $[128,129,130]$.

\subsubsection{Discussion}

In order to define the best sizing architecture of the RES used, different approaches can be used to supply the user's demand following their requirement for optimization. None of them guaranties the success of the sizing process. To be sure to find an appropriate sizing architecture, an interesting approach is probably to cross several methods and to compare the obtained solutions. The sizing process however not only depends on the future usage but also on the future weather conditions that have a high level of uncertainty. Reducing this uncertainty is till nowadays not possible and robust optimization approach to address this problem could appear as a promising perspective.

\subsection{Energy-related Standards and Metrics}

While energy efficiency metrics for datacenters, such as Power Usage Effectiveness (PUE), have been in use for years, the integration of renewable energy requires defining new metrics. Normalization bodies have been working for several years on this issue. In particular, ISO (International Standardization Organization) and IEC (International Electrotechnical Commission) built a joint committee (ISO/IEC, JTC1/SC39) in order to propose some normalized metrics. A series (30134-*) of normalization documents have been proposed since 2016. Standardized key performance indicators exist, while other are still being discussed. The datacenter perimeter for assessing the energy efficiency is defined in document 30134-1. Within this perimeter, PUE is defined as the ratio between the energy supplied to the datacenter and the energy used for the IT equipment. Renewable Energy Factor (REF) represents the percentage of renewable energy in the total power used by the datacenter. Finally, ERF corresponds to the percentage of energy reused from the heat generated by the datacenters.

Researchers have proposed other metrics that will have to prove their interest and wide acceptance before going to normalization. Several European projects on energy-efficient datacenters, e.g, CoolEmAll [131] and RenewIT [132], have evaluated such metrics. A cluster of projects working on renewable-powered datacenters published in 2014 an evaluation [133] on relevant metrics. One of the main conclusions is the heterogeneity of the metric field, leading 
to the need to measure too many metrics for assessing datacenters. The proposed overview encompasses more than 40 different metrics, classified in four categories, and selects one metric in each category to act as a representative. The selected categories are Basic energy efficiency, Cost-environmental analysis, Grid interaction and finally Capacity metrics. In [134], these metrics along with their mathematical formulation are provided.

\section{Management of Green Datacenters}

In the previous section we consider the design of the energy infrastructure. Since the design of the IT infrastructure in a datacenter powered by renewable energy does not imply specific challenges compared to the design of a standard datacenter, this issue is not tackled in the paper. In this section we focus on the infrastructure management, both from an energy and an IT point-of-view. The infrastructure management addresses the dynamic part of the challenges to be tackled when powering a datacenter with RES. Note that the literature on IT management is much larger than the one on energy and is a very active research domain. When trying to manage both the energy and the IT platform, most methods are approximate, and often based on greedy or evolutionary algorithms. As stated below, each problem is complex, and managing both leads to the same NP-complete complexity. Even when the management of the whole infrastructure is based on the cooperation of independent IT and energy managers [135, 136], the cost is the sum of both, adding the cost of multiple round of negotiation between them.

\subsection{Management of Energy Sources and Storage}

In order to use the renewable generation and storage units in the most adequate way, energy management strategies need to be designed and implemented. Haddad et al. have proposed in [137] to solve several storage management problems considering different objectives (best constant power production, maximization of the level of hydrogen at the end of a given time horizon, etc.) using a mixed integer linear programming approach to take numerous constraints imposed by electrical models into account. While it is possible to control the output of RES up to a certain point, mostly to decrease it, it is usually preferable to control the operation of energy storage units to have generation and demand match in real time. Control strategies can follow a variety of objectives (e.g., minimizing costs or emissions [138], peak shaving [139]) and constraints (e.g., power balance, power limits, state-of-charge limits, ramp-rate limits [140], or stability), while different types of models (e.g., transfer functions or static models based on simplified physical behavior) may be used depending on the intended application and the related time constants. This topic has been extensively studied in the literature in the general case of power systems and microgrids, such as in $[141,138,142,143]$ however the interest for datacenter applications is rather low since most contributions for microgrids could also be applied to datacenters.

Nevertheless, there have been recently several studies that consider energy sources management specifically for green datacenters. In [144], Deng et al. try to decrease the operation cost of datacenters by introducing an online control policy that uses several characteristics of several energy sources. The authors use Lyapunov optimization and design a control algorithm that does not require significant statistics on the dynamics of the system. The control algorithm makes decisions based on long-term ahead power procurement, real-time power procurement, and realtime UPS charging/discharging. An one-month trace-driven simulation shows that the proposed algorithm provides a compromise between operational cost, UPS lifetime and datacenter availability. The algorithm is also robust to time-varying supply and power demand. A second paper concerned by the management of many power sources to supply autonomous datacenters is [145]. Li et al. worked on green datacenters powered by multiple power supplies such as basic, intermittent power, and backup power storage. The authors notice that existing studies generally assume some specific variations of RES and neglect the benefits of the entire system integration. They hence consider a "hierarchical power coordination system" that manages the capacity of the RES and when to use them. As a result, datacenters ensure multi-objective energy management decisions, based on renewable energy variability, base-load power availability, computer tasks, and storage capacity.

The paper [146] also considers the coordination among multiple power sources in datacenters, but mainly focuses on experimental research. The sources are scheduled considering a priority scheme, the objective is to maximize the utilization of renewable energy, while minimizing the power consumption from traditional grid, and optimizing the battery operation. The renewable energy, grid and batteries are assigned with first, second and third priorities, respectively. A dynamic power technique is employed to cap the energy used from the grid. In the simulation, the renewable source is solar energy. The approach in this paper can also be categorized as joint management of IT and electrical infrastructure, since the authors claimed that a workload scheduling algorithm is implemented in the 
Table 1

Summary of Energy Management Systems (EMS) for green datacenters

\begin{tabular}{|c|c|c|c|c|}
\hline Ref. & $\begin{array}{l}\text { Stand- } \\
\text { alone }\end{array}$ & $\begin{array}{l}\text { Grid } \\
\text { con- } \\
\text { nected }\end{array}$ & System configuration & Energy Management \\
\hline [144] & & $\checkmark$ & $\begin{array}{l}\text { Renewable energy/ UPS batter- } \\
\text { ies }\end{array}$ & Lyapunov optimization \\
\hline [145] & & $\checkmark$ & $\begin{array}{l}\text { PV/wind/batteries/Fuel Cell } \\
(\mathrm{FC}) / \text { Gas turbine }\end{array}$ & $\begin{array}{l}\text { multi-objective power man- } \\
\text { agement decisions }\end{array}$ \\
\hline [147] & & $\checkmark$ & PV/Wind/batteries & $\begin{array}{l}\text { holistic approach that re- } \\
\text { duces electricity cost and en- } \\
\text { vironmental impact }\end{array}$ \\
\hline [148] & & $\checkmark$ & CHP plant / FC & $\begin{array}{l}\text { optimization of energy con- } \\
\text { sumption operating costs } \\
\text { and } \mathrm{CO}_{2} \text { emissions }\end{array}$ \\
\hline [149] & & $\checkmark$ & Wind/batteries/Supercapacitors & $\begin{array}{l}\text { heterogeneous energy } \\
\text { buffering power approach }\end{array}$ \\
\hline [71] & $\checkmark$ & & PV/Wind/hydrogen & $\begin{array}{l}\text { developed search algorithm } \\
\text { to find LCE that meets zero } \\
\text { LPS }\end{array}$ \\
\hline
\end{tabular}

simulator. However, the IT scheduling is not discussed in detail, and the coordination between IT scheduling and power sources scheduling is not provided. Another approach is presented by Liu et al. [147] which models the energy flows in a datacenter and optimize their usage. While, the availability of cooling or energy, like other supply-related constraints, is usually treated separately from the management of IT work, their work reduces the environmental impact as well as the electricity costs while using a holistic approach. The latter integrates dynamic pricing, renewable energy supply, cooling and workload scheduling to secure the sustainable operation. Renewable energy and computer demand are predicted, and these predictions are used to generate IT workload management capable of scheduling the IT workload while taking into account the efficiency of power and cooling. Using this two-phase technique, authors show a reduction of the usage of grid electricity by $60 \%$ without a negative impact on the quality of service of the applications. Similarly in [148] the authors consider a datacenter with a power consumption of $100 \mathrm{~kW}$ originated from the IT part. It includes the values of energy efficiency specific to this infrastructure. The authors aim at increasing the energy efficiency of the datacenter management system. The approach is completely based on a direct current (DC) architecture. It uses high performance chillers and a power supply from a Combined Heat and Power (CHP) plant (aka co-generation), instead of the power grid. Executed simulations using advanced energy management technologies deliver significant energy, cost and emission savings when operating a datacenter.

Another approach for managing power supply in green datacenters is power provisioning, as in [149]. In that paper, Liu et al. consider the datacenters that may be supplied by under-provisioned or intermittent power. The authors claim that devices such as an UPS cannot handle in a efficient way the irregular and unpredictable power mismatches. They propose a power provisioning architecture called Heterogeneous energy buffering (HEB) that supports the simultaneous usage of Super-Capacitor (SC) and batteries. Compared to homogeneous storage technologies, the heterogeneous approach needs more intelligent power management to exploit the capacity of different energy buffers. The authors propose to assign dynamically the workloads among SCs and batteries. The goal is to achieve a better energy efficiency, while maintaining a low performance degradation in case of power mismatching. The experimental results from a real system prototype show that, compared to HEB design with a homogeneous battery energy buffering approach, HEB can improve renewable energy utilization, increase UPS lifetime, and decrease system downtime. Similarly, Iverson et al. [71] present a search algorithm that aims at giving the minimum Leveled Cost of Electricity (LCE) that meets zero Loss of Power Supply (LPS) in the context of datacenter application. They design a model comprised of several subsystems. This model is used to assess two scenarios depending on the control ability of the demand.

\section{Discussion}

From the strict energy management point of view, a datacenter is a system that has to be fuelled as any system with 
a given energy demand. On the other hand, introducing renewable energy in the energy supply implies to consider the datacenter as a global system that contains both an IT part and an energy supply part. As renewable energy is intermittent by nature, different problems have been addressed in the literature with different objectives so as to reduce sometimes the economical or the environment impact of the datacenter. Constraints of these problems differ from one problem to another. Moreover, as managing microgrids is similar to managing the electrical part of the datacenter, improving the management of the energy sources and storage in a datacenter context leads to address new challenges, as for instance, reducing the energy consumption by using new IT scheduling approaches or synchronize as much as possible the energy demand and production (for instance decrease the datacenter consumption in case of power shortage by switching off servers, delaying tasks, etc.). Furthermore, since renewable energy remains intermittent with a certain uncertainty, managing energy of the datacenter also often means managing storage devices, as SC, batteries or other energy provisioning devices depending on whether it is for short term or long term storage. The optimization of the usage of the power grid connection is also an option. Papers in the literature shows that managing energy sources and storage is all the more efficient when the different parts cooperates.

\subsection{IT management}

In this section we present research works tackling the issues related to the IT management when a datacenter is fuelled by RES. In that case, the problem is to adapt the IT resource consumption to the energy or power availability. Two leverages can be used, server shutdown or suspend and workload management, both based on scheduling or load balancing, with the considered workloads that can be composed of batch jobs, services or both. The methods used by the cited works to manage or adapt the IT consumption are: job scheduling, virtual machine migration, service rate allocation, software controlling, shifting demand in time, assigning IT jobs to computational resources, and DVFS. Table 2 gives a synthetic view of the method used for each of works described below. The objectives are to minimize criteria like the finish time of a job, or of a set of jobs (makespan), to maximize SLA or service rate while minimizing brown energy consumption or remaining in a power prediction. Note that, as the classical scheduling problem (i.e. without constraint and with only the decision of when and where to run applications) is NP-complete[150], scheduling in the context of renewable energy and with a large number of leverages reach the same complexity. Most approximation algorithms are based on greedy algorithms, which are often at most quadratics or evolutionary, which are usually with a polynomial complexity. Some of them are based on mixed integer-linear programming methods to explore small scale systems, using solvers that are exponential in the number of integer variables in the worst case. A batch job does not run interactively but is rather submitted to a batch scheduler that decides when and where to run the job. This kind of job is submitted with an expected end of execution time which can be a hard constraint (called deadline) or a soft constraint (called due date). To control the power consumption of the workload, the batch scheduler can delay a job in time or control the voltage and frequency of the processor it is assigned to, for instance to shift or decrease a power consumption peak to respect a power capping, or it can group the jobs on the hosts to power down some of them.

A service is an application that handles requests issued by interactive clients, as for instance a web server. A service is characterized by a service rate, the number of handled requests per seconds, and the amount of resources consumed to process these requests. Power consumption of a service can be limited by decreasing its service rate. In that case, limiting the resources allocated to the virtual machine may decrease the service energy consumption and migrating virtual machines to group them may be a way to power down some servers.

In the following we first present works that consider only one datacenter, which means that RES, usually supplemented with an electricity grid supply, are globally managed. The characteristics of each contribution are summarized in Table 2. We then present works that consider several, distributed, datacenters, which means that several sources of energy and/or power availability are separately managed. The characteristics of the contributions are summarized in Table 3. In each of these parts, we distinguish the works that either consider batch jobs, services or both.

Note that this survey is limited to works that consider RES and do not consider energy consumption reduction. In addition, since the article of Kong and Liu [23] already surveys works on renewable energy and reduction of carbon emission before 2013, we also limit this study on works that are not included in this article or that have been published since 2013.

\subsubsection{Single Datacenter Approaches}

As previously said, considering only one data center implies that the RES are globally managed.

\section{Approaches dealing with services}


Several research works consider the IT workload as services and their objective is to maintain a service rate while maximizing profit or efficiently using the available green energy. Note that the service rate approach allows to use a continuous objective and thus it is possible to define linear programs to optimize the problem solution.

Ghamkhari et al. [151] consider service rate allocation in green datacenters with local renewable power generation. The authors consider a trade-off between maximizing the datacenter profit and minimizing their energy expenditure. The profit is defined to be the difference between revenue and cost.

The research in [152] uses the approach of virtual machine migration. They propose a green-aware power management strategy, taking into account the consumed electricity of both cooling and functional infrastructures. The goal is to use green energy and maintain the consumed power at an acceptable level. An optimization problem of virtual machine migration is formulated and solved using statistical searching and heuristics.

\section{Approaches dealing with batch workload}

With a batch workload approach, the load cannot be modeled by a continuous variable since it is more coarse grained. The proposed solutions are closer to classical scheduling algorithms. Different approaches, listed in the following, tackle variations of the problem.

Most of the studied works tackle the problem of scheduling the workload to optimize the renewable energy use while minimizing the grid inputs. This is the case in several papers from the research of Goiri et al. In [153], the authors introduce an energy management algorithm for parallel task scheduling, considering photovoltaics and grid power. The scheduler aims to maximize the renewable electricity utilization while minimizing the cost of electricity consumption of the datacenter. A grid connection is used as a secondary power supply when the solar generation is too low. A controller schedules the tasks on the low cost hours in order to reduce the electricity purchase. In [154], the authors propose a two-level control method to solve a multi-objective optimization problem in a datacenter. While reducing the deviation between reference and actual power profiles at the upper level, the algorithm increases the quality of service at the bottom-level using the received power profile. The reference consumption profile is predetermined by taking into account the expected renewable generation. Using the Pareto-front optimal solution, the developed control algorithm finds the best control strategy that maximizes the green-energy consumption.

In [155], Lei et al. also develop a task scheduling strategy for a green datacenter powered by both electrical grid and renewable energy. The energy from the grid is used depending on the renewable energy prediction and the dynamic electricity pricing of the grid. Lei et al. [156] use DVFS for handling IT consumption. DVFS is usually applied when a slack period is detected, then the processor can run at lower frequency or can operate on lower voltage, which decreases the power consumption. To do so they propose a multi-objective evolutionary algorithm.

In the same context, but in an approach that takes into account the forecast of renewable energy and batteries, in [157] the authors propose a scheduling algorithm based on priorities. They introduce a sizing methodology, in which the amount of solar panels and batteries are formulated as a function of the expected workload. A genetic algorithm computes the task allocation, using the available RES and storage capacity. The overall decision on the electrical infrastructure depends on the IT scheduling decision under the constraint of available renewable energy.

Still taking RES and grid as power supplies, authors of [136] introduce an interaction between the power supply and the batch scheduler. They propose a scheduling algorithm with two objectives to minimize, the quality of service (due-date violation) and the grid power (non-renewable) consumed. The electrical infrastructure is considered as a black box with its own optimization process. Contrary to the classical centralized optimization of both electrical infrastructure and of task scheduling, the batch scheduler only uses a minimalist communication model where the amount of information coming from the electrical black box is reduced to a simple positive or negative feedback.

Also taking the power supply into consideration, the authors in [158] present a global algorithm optimizing at the same time IT jobs and the physical infrastructure of datacenters, including the computing and cooling elements. The proposed approach minimizes several objectives, including energy consumption, by minimizing the distance between a target power profile and the planned one.

In a different context, Klingert et al. [159] studied a RES-powered datacenter with workload coming from smart cities. The sensor networks in a smart city collect data and store them in a datacenter. They propose technical and business approaches to manage the local RES. To do so, the authors introduce adaptation strategies together with power management options. Based on the availability of RES and the state of the grid, the datacenter schedules the IT tasks, virtual machines and servers accordingly.

Other approaches consider that IT resources are solely powered by RES. The research from Kassab et al. [160], [161] considers the problem of scheduling independent tasks under power constraints, defined from a power prediction from 
RES. In [160], several common standard scheduling techniques such as list scheduling and binary search are adapted to take power constraints into account. Their approach does not aim to reduce the energy consumption, but rather to optimize scheduling objectives such as minimizing the makespan or the total flow time, while respecting the power constraints given by a power prediction. In [161], a genetic algorithm is used to deal with the IT job scheduling problem in green datacenters. The paper assumes that different tasks have different power consumption, according to their computational density; therefore, changing the order of the tasks leads to different power consumption levels at a given time. In [162], the authors introduce an IT optimization module for datacenters powered only by RES. The modules processes batch workloads which are also scheduled under renewable energy prediction, but the objective is to minimize the number of due date violations.

\section{Approaches considering a mixed workload}

Datacenters are seldom used for only one type of workload. We have grouped here works that consider both service and batch workloads or do not differentiate between workload types. In this context, the optimization of the renewable power use remains the main concern but optimization objectives as quality of service are preferred to other objectives such as the makespan. We present in the following the characteristics of the different surveyed works.

The authors of [163] study the approach of software controlling, which allows to adapt the power needs of applications to the power availability. They introduce a generic framework and software controller named Energy Adaptive Software Controller (EASC) which enables applications to react to the intermittent nature of renewable power production. This framework applies different degradation modes to the applications, from none to deeply degraded. EASC manages all the applications and their degradation levels to optimize the overall quality of service in a power prediction.

In [164], the authors consider simultaneously job spatial scheduling (on the appropriate servers), job temporal scheduling (allocating the jobs in time), VM suspend/resume switching, and VM migration. A proposed heuristic algorithm schedules jobs spatially, temporally, and a VM controlling mechanism manages the VM switching and migration. The authors introduce EpoCloud, a prototype that optimizes the energy consumption of mono-site datacenters powered by the electricity grid and RES. The objective is to find the optimal trade-off between the cost of energy and the QoS degradation. In [165], the authors also consider a temporal load balancing problem, but with a demand response approach. The authors provide intensive analysis about the potentials of demand response in datacenters and recommend to use the pricing approach that is based on the prediction of workload demand and renewable generation.

The workload in [166] is not clearly stated to be batch or service, but instead "real-time" and "delay-tolerant". The authors consider a straightforward approach that shifts the energy demand of the datacenter in time, from the time with less available RES to the time with more RES. The objective is to increase the utilization of on-site generated renewable energy. The shifting is realized based on the flexibility of electrical cooling systems, IT workload, and diesel generators. In [167], they consider real-time delay-sensitive jobs (services) and deferrable jobs with due dates. The problem is viewed from the perspective of demand response, which is a spatial/geographic load balancing problem. The authors propose a straightforward strategy for shifting in time the due dates of IT jobs, while providing monetary incentives on deadline deferral, in order to match the renewable energy availability.

In [121], the authors introduce two approaches to improve the utilization of renewable energy in datacenters with power storage. The first scheduler keeps some low priority jobs ready to be executed when renewable power is in excess. The second one stores the renewable electricity surplus and uses it later when renewable energy is scarce. The objective is to maximize the utilization of renewable energy produced on-site.

The interest of research presented in [168], [169] is that they focus on building a practical research platform to study green datacenters. The authors introduce Parasol, a prototype of a green datacenter with solar energy, batteries, and net metering. Additionally, the authors introduce GreenSwitch, a dynamic scheduler of power sources and workload. In [169] the GreenSwitch scheduler tries to reduce the grid electricity costs, with regard to the workload characteristics and the battery lifetime. However, in [168] and [169] only solar panels are considered as RES and the IT scheduling is limited; specifically, the authors use scheduling algorithms only to select the energy source to use (renewable, battery, and/or grid), and choose the storage medium (battery or grid) at each time period.

Last, the approach of the DataZero project [170], [162], [160] is based on a joint IT and energy management in a green datacenter that is entirely powered by renewable energy. The authors propose a system with one standalone microgrid and three different management modules for IT, power and negotiation. The scheduling strategies of both IT and power are first considered locally by the IT module and the power module then a trade-off between both solutions is considered jointly with the support of a negotiation module. This module coordinates the power demand and power supply from IT side and electrical side. 


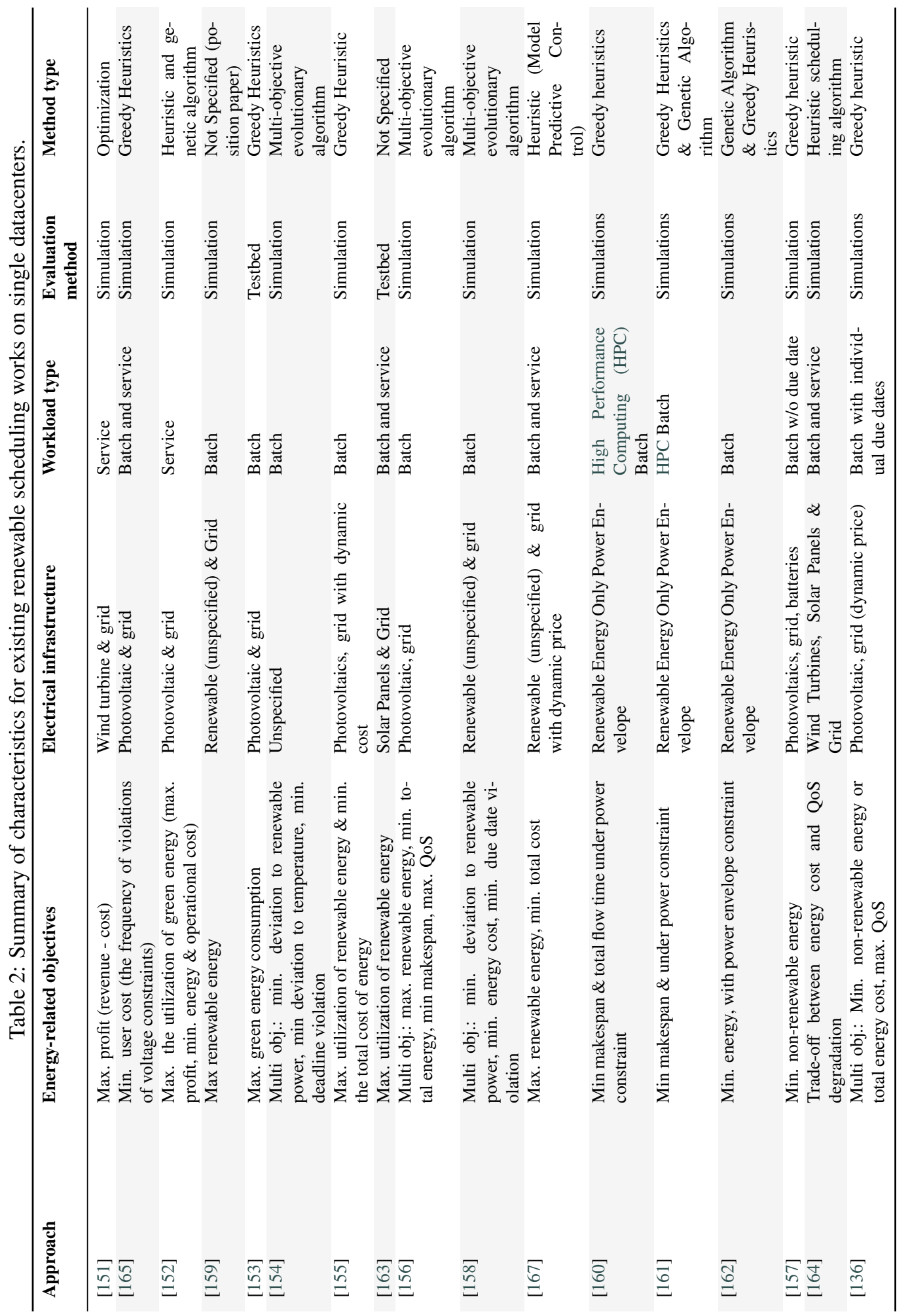




\section{Discussion}

Table 2 summarizes the main studies, cited in this section, on the design of a single datacenter with RES inputs. The table synthesizes, for each publication, the energy related optimization objective, the energy sources, the workload type, and the approach used in the study. As we can see, most of the works aim to increase as much as possible the green energy consumption or to decrease the cost of using electricity from the grid and only two works consider powering the datacenter with only RES. Similarly, most of the works use a greedy algorithm or a genetic algorithm to search for a good solution while only one article uses a linear programming approach. This choice is understandable since the problem is complex when we consider a non continuous workload as the latter problem is based on a NP-Complete parallel scheduling problem. Last, it is unfortunately not possible to compare these works with each other since they do not target the same objectives and do not use the same workload.

\subsubsection{Energy management in multiple datacenters with renewable energy}

This section presents studies on IT management in multiple geographically distributed datacenters. Taking multi datacenters with local RES into consideration in the problem of managing the IT workload allows to balance the workload according to the available energy/power that may be different on several sites. In that case, Graphical Load Balancing (GLB) is widely explored, as presented by Rahman et al. [184] in a comprehensive survey on several approaches with and without the usage of renewable energy and storage elements. In this work, we focus only on GLB with renewable energy, giving emphasis to the more recent approaches.

\section{Approaches considering service workload}

Since both renewable energy availability and the workload are expected to differ from one datacenter to another, migrating some $\mathrm{VMs} /$ containers/tasks from a datacenter with low local renewable energy production and high workload demand towards another datacenter with renewable energy surplus, reduces the need for brown energy. The initial works that explore the use of GLB and renewable energy are [171] and [172]. Liu et al. [171] evaluate feasibility of powering cloud datacenters with renewable energy. The authors focus on GLB, using a concept called "follow-therenewables", that migrates the workload between datacenters powered by renewable energy. The goal is to improve the renewable energy usage considering the different locations and weather conditions. Lin et al. [172] present an extension of the previous work with online algorithms to exploit the geographically distributed datacenters and their renewable energy availability.

Some more recent approaches aim to minimize the carbon emission such as [176], and/or reduce the price of the energy acquired from the grid such as [177] and [178]. In [176], the authors tackle the problem of IT job management by dynamically distributing the computational requests to the RES-powered datacenters. They model the problem as a constraint optimization problem with the objective to minimize carbon emissions with a fixed electricity budget. Another research from Gu et al. [177] considers datacenters powered by wind turbines, solar panels and energy from the power grid (brown energy). The authors also use storage devices to buy energy at low prices (electricity prices vary depending on time and location) or store renewable energy surplus. The work focuses on two optimization problems: (i) to respect the QoS demand and minimize the energy cost; and (ii) to minimize the total carbon emissions under an energy budget. [178] focus on online algorithms that take decisions at run time for each incoming task. They aim at minimizing costs, exploiting the variations in the grid prices and the renewable energy utilization, also in a scenario with several datacenters. The authors explore the price volatility, leveraging from the agreements in the electricity market, anticipating how much energy should be bought.

More recent works, that focus on maximizing the renewable energy usage, as in [180] and [181] also consider GLB to maximize renewable energy utilization and consequently reduce energy expense. [180] focus on practical considerations and present experiments using real machines in Grid5000, traces of English Wikipedia traffic, renewable energy, and electricity price traces. The authors consider two load balancing levels, local in a datacenter, and global to distribute the load among different datacenters. [181] propose to migrate VM between datacenters, located within the same region, to maximize the consumed green energy, with a simplified model (high level view of the datacenters rather than machines, processors...). The authors present three different approaches depending on the usage of an oracle knowing the future of renewable energy availability.

Other works such as [175] and [179] focus on different constraints, or metrics. Zhang et al. in [175], also tackle the problem of minimizing the brown energy usage using virtual machine migration depending on the availability of renewable energy, but they add network capacity constraints, considering more specifically Elastic Optical Networks 


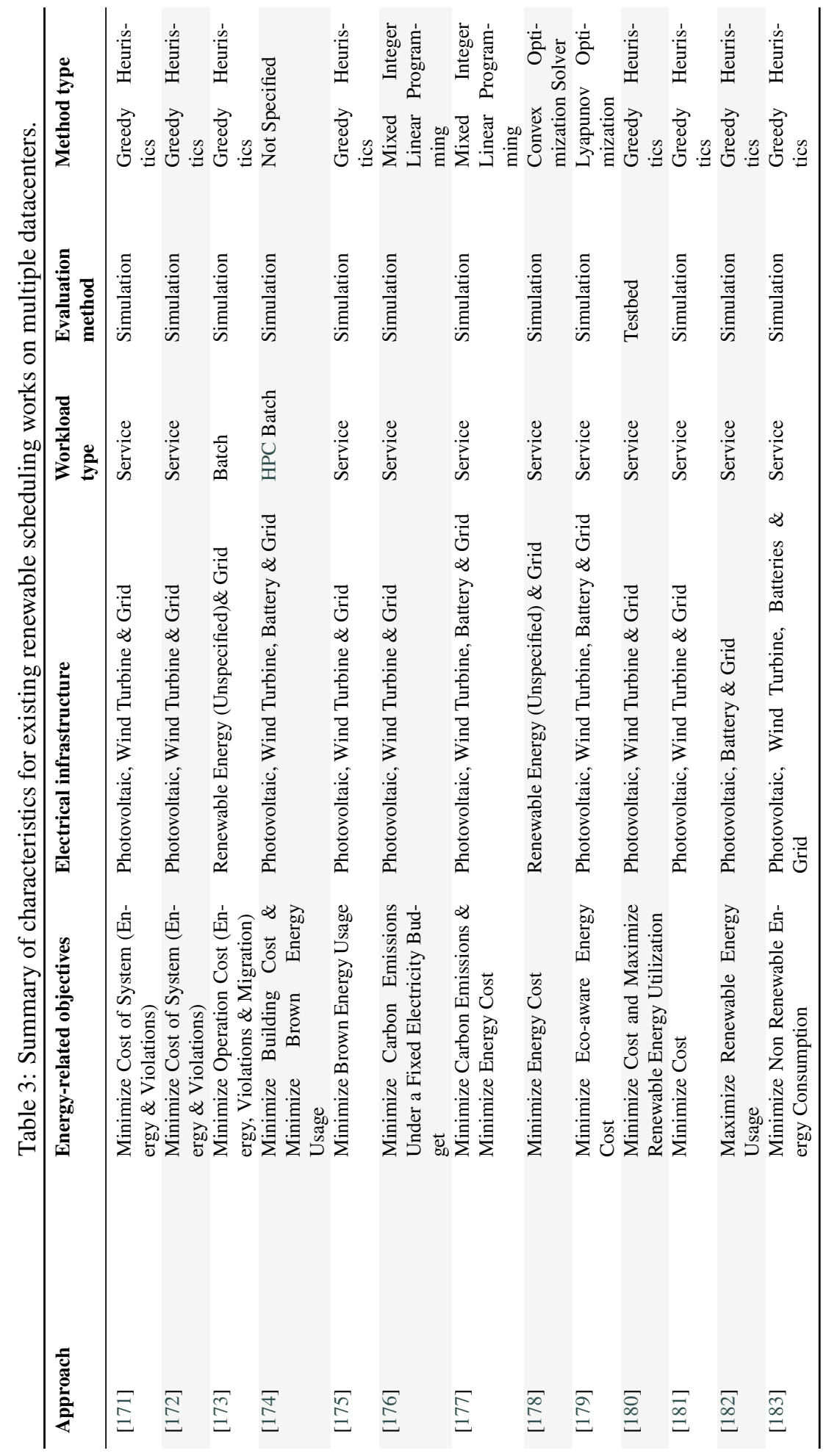


(EONs). They propose two heuristics taking into account the influence of the network capacity. Deng et al. in [179] focus on minimizing the eco-aware energy cost, different from the normal cost, by assigning an eco-factor parameter to each type of power supply (power grid, energy storage system, solar and wind powers). Eco-factor refers to a level that indicates the green degree of the utilized energy from every supply. The parameter is determined according to the environmental impact of each supply during the energy production (grid power) and equipment manufacturing (green resources).

In more recent works such as [182] and [183], the authors use the same concepts but introduce the usage of containers and edge computing. Li et al. in [182] propose to leverage renewable energy production of edge nodes. The authors propose an analytic model in order to decide if the computation should be offloaded from the connected objects to the edge or directly to the Cloud according to the renewable energy availability and the Quality of Service requirements. The works rely heavily on on-site renewable energy production and storage at the edge nodes. Aujla et al. in [183] propose an energy-aware job classification and scheduling scheme that uses a Container as-a-Service (CoaaS) approach to enable the applications to be balanced depending on the local energy production of each datacenter.

\section{Approaches considering batch workload}

There are a few studies ([173] and [174]) that address the batch workload in multiple distributed datacenters. Goudarzi and Pedram in [173] focus on the load balancing of batch jobs with dependencies in heterogeneous datacenters. The authors aim at reducing the cost considering RES and energy pricing variations. The proposed approach is an online greedy scheduler where each datacenter has limited resources and maximum generated power. Berral et al. in [174] focus on studying the location and cost of provisioning several green datacenters and renewable plants, that provide a given level of renewable energy on-site (the rest comes from the grid), for a "follow-the-renewables" approach.

\section{Renewable energies and Cooling}

Cooling accounts for an important share of the electricity costs for a datacenter. Going further than only considering cooling as an additional electrical load, some researchers focused on the link between cooling and location of the datacenter, especially in the presence of renewable energies. H. Khalaj et al. [185] studied 42 locations to assess their production and free cooling potential and gave results on optimal sizing for hybrid power infrastructure, opening the path for a "follow-the-renewable" approach taking into account the cooling. Additionally, anticipating the cooling of a datacenter according to the availability of energy (aka precooling) has been investigated by Zhang et al. [186] and Lukawski et al. [187], where authors consider energy reduction as a financial operational cost to minimize. Li et al. [188] and Madon et al. [189] covered synergies between renewable production and pre-cooling possibilities for the maximization of RES, depending on the location of the datacenter.

\section{Discussion}

With the same characteristics used in Table 2, Table 3 summarizes the main studies tackling the management of multiple datacenters cited in this section. We can note that, considering distributed datacenters, the cost objective is more often used than for a single datacenter. Actually, this is almost the same as maximizing the renewable energy consumption since the green energy is generally considered as free when it is produced on site. On the other hand, these studies more often use several objectives to balance between energy consumption and quality of service. Last, as with the single datacenter works, greedy heuristics are mostly used as a solution to improve the objective. Note however that exact methods as linear programming seem also be a possible optimization approach since they are more used in this context.

\section{Conclusion and Open Research Challenges}

This article presents a survey on datacenters powered by renewable energies and the related issues. Its objective is to synthesize the research works done around this idea of electrically green datacenters. We thus consider the linked issues at different levels, first the whole datacenter and then its composing parts: electrical infrastructure, energy management and, last, IT management. The integration of RES has been thoroughly investigated, as proved in this survey. On the other hand, after surveying all these works, our conclusion is that most of the research works tackle only a part of the datacenter and propositions addressing the issues of designing a whole datacenter of, at least, medium size (i.e. from the sizing of the power sources and storage to the respect of the SLA) are lacking. To reach this goal several open research challenges remain to be tackled. We present some of them in the following. 


\subsection{Infrastructure challenges}

Different challenges could be addressed on the infrastructure of datacenters: first redundancy could be addressed in a different way and second cooling could be provided with storage equipment. In a classical datacenter architecture, redundancy is a first choice to overcome the risks of having only one electrical provider or to allow switching machine rack power distribution from line A to line B. Disruptive architectures taking into account reconfigurable power distribution systems have never been addressed. Energy storage in a number of hydrogen tanks and batteries can play an active role as backup sources in addition to the primary sources (solar and wind) within a real time dynamic, reconfigurable power distribution system in the datacenter. It would improve service continuity and reduce costs without sacrificing high availability, in particular in the presence of geo-distributed datacenters. Datacenter cooling is studied in the literature in different ways; for example thermal-aware scheduling and free-cooling approaches are studied. However fuel cells and hydrogen could offer some possibilities to reuse heat to cool the datacenters. Fuel cells and hydrogen have been studied for storing energy and producing power for the datacenter, including the IT equipment and the cooling. A novel option would be to reuse the heat generated by the fuel cells and transform this heat to cold.

Finally, an aspect that has been neglected in previous works considering renewable energies and datacenters is the total ecological footprint [190]. Integration of the Life Cycle Analysis (LCA) aspects in the decision-making algorithms (IT scheduling, power sources commitment) should be addressed. To do so, easy to assess metrics should be used to compare the usage of different technological possibilities, and to assess at best the potential obsolescence of the equipment and their total ecological cost of ownership, beyond the total cost of ownership.

\subsection{Electrical challenges}

Most of the existing literature so far has focused on supplying datacenters with RES or improving energy efficiency. However, grid-connected datacenters can also provide ancillary services to the grid [191, 192, 193], i.e., they can absorb or supply power on short notice and participate in frequency regulation or reserves, for example. Such behavior can be obtained either by modulating the IT load [194] or using local storage resources, e.g., from existing UPSs or from supplementary storage units [195]. This can generate an additional stream of revenue for datacenter operators [196], as such services can be sold on markets or through long term bilateral contracts. This does however also complicate the management of resources, and requires the use of portfolio optimization techniques [197].

While most approaches using storage focus on coupling such units at datacenter level, some works have also investigated using storage devices at server level. In [198], authors study combining batteries and/or supercapacitors with each server for peak power shaving and capping. Several control algorithms are tested, and results point towards the usage of supercapacitors. The research shows that supercapacitors can reduce the total cost of ownership (TCO) by $34 \%$ compared to a case with batteries only. However, multiplying the number of storage devices makes maintenance more difficult.

Another aspect that would deserve further investigation is the aging of components and its impact on resource management and sizing. As mentioned earlier, degradation effects tend to decrease the performance and efficiency of components and their ability to absorb or supply large currents. Aging models are however not mature enough or far too complex or impractical to enable accurate prognostics of performance degradation for use with maintenance. Further research is therefore needed in this area, which could reduce the occurrence of unexpected over-costs linked to early component replacement.

Last, but not least, designing and using new energy sources (such as bio-methane) and storage solutions is a worldwide challenge. For the storage solutions we can cite the use of kinetic energy as proposed by Energy Vault [199] or heat as done with the thermal energy devices of CCT [200]. Each new source or storage comes with its own constraints and will imply the design of appropriate models and infrastructures to integrate them in tomorrow's datacenters.

\subsection{Prediction and online decision challenges}

One of the main challenges remains uncertainty in the workload and in the power generation, since both IT and electrical optimization highly depend on the input data. One way to address this issue is to predict these input data, the workload for the IT part and the energy for the electrical part. This would allow to turn the online optimization problem into a static optimization problem that usually gives better results.

For the electrical part, weather predictions are already used to anticipate the energy production by photovoltaic and wind turbines but advanced prediction techniques like deep learning [201] may improve the accuracy of the prediction. For the IT part, having an accurate prediction of the coming workload will allow to better anticipate the IT management. 
For instance, knowing that the load will probably increase allows to be anticipated by powering on more machines and cooling elements and ask for more energy on the electrical part, for instance activate a fuel cell.

Concerning the IT load prediction, another challenge could be to predict the energy consumption of a task, given its input data. There is already a large set of research works that tackle the prediction of the running time of a task, given its parameters or input data, but we could also consider predicting its power consumption over time, in time slots. This will give a more accurate information to the scheduler that could better use the available power in each time slot. Such prediction may be based on Deep Learning.

Another way to tackle this uncertainty challenge is to study online decisions between the IT power consumption and the electrical power production with multi-source commitment. The robustness of optimization algorithms in presence of uncertainty should also be studied. This would adapt dynamically the schedule for IT and sources commitment for power sources. Based on the current new situation (more or less power needed, more or less power availability), reactive actions could be proposed (re-allocation of tasks, QoS degradation, local command on power sources...). This on-line reactivity should take into account uncertainties. Reinforcement learning should help to understand the effects of cumulative decisions previously taken and improve future ones.

\subsection{IT challenges}

To the best of our knowledge, there is until now no evaluation of feasibility nor performance of geographically distributed datacenters powered only by renewable energy (no grid as backup). This kind of scenario can be specially interesting considering very distant datacenters (different timezones).

One main open problem concerning renewable-powered datacenters comes from their assessment. To be able to evaluate their performance, it is necessary to use either actual infrastructure or simulation. As the first one is impractical due to the size and the hardware complexity, the second one is mandatory for large scale tests. This leads to two open challenges:

- Co-simulation: Ooe main difficulty concerns the large number of necessary simulators from different fields, from IT simulators to energy-related ones;

- Complete traces: some long-term traces exist for particular datacenters or for renewable-related elements (wind, irradiance, temperature, ...) on particular places. At the moment there is no reference dataset encompassing these two worlds. Plus we are missing large sets of power consumption by application vs processor or, better, hardware architecture.

\subsection{Simulation and validation challenges}

The cost and time required for performing experiments on large-scale datacenters are usually high. In addition, such experiments are difficult to reproduce, due to their expensiveness as much as due the difficulties to access such infrastructure. Simulation is therefore a valuable tool to design and evaluate grid- and cloud-computing scheduling approaches. It may consist of ad hoc numerical simulations, or in using existing simulators developed by the community, depending on the complexity of the model, the target of the study, and the required degree of accuracy. Several simulators are commonly used in the HPC and Cloud Computing communities, such as SimGrid [202], CloudSim [203] and DCworms [204].

When RES are considered in datacenter management approaches, it may be required to simulate some of the behaviors of the electrical infrastructure itself. The immediate or predicted power production of each renewable source may be required to take some decisions. Such data are easily added to existing simulators, as they may be pre-computed offline based on real production traces or weather conditions and source models. However, when energy storage devices are also considered, deeper integration with the simulation tools is required. Indeed, the energy stored at a given time must be updated based on the power consumption of the computing part and the available power.

Simulating a datacenter with RES requires at least two kinds of data: the workload of the datacenter itself and the electrical production of the sources. To evaluate an approach using simulation, the workload may be either based on real-world traces or generated using known properties (synthetic workload). Examples of publicly-accessible realworld traces are the Google cluster data [205], analyzed by many authors [206, 207, 208], the different traces available on Grid Workloads Archive [209] or the traces of user requests on the 1998 World Cup [210]. Depending on the context of each approach, the workload model may differ significantly, with the two main categories being batch tasks and long-running services, as detailed in Section 3.2. Therefore, all the available workload traces or synthetic generation methods do not fit the requirements for every study. 
From the other side, the electrical production of the sources is calculated based on models and meteorological data such as wind speed and irradiation. These data could be obtained from various databases online such as the national solar data base (NSRDB) [211], the Modern-Era Retrospective analysis for Research and Applications (MERRA2) [212], the wind prospector from the National Renewable Energy Laboratory (NREL) [213].

None of the general purpose datacenter simulators are distributed with the ability to simulate such RES and storage devices. A few works already attempted to integrate these features, such as [136] in which authors extended DCworms with electrical infrastructure simulation. But there features were not used by other authors of the community until now. Therefore, almost all the works targeting datacenters with complex electrical infrastructures use ad hoc simulators written specifically for their needs [214, 215, 121].

Performing experiments with real hardware is even more difficult when RES are studied. In addition to demanding expensive components, the high unpredictability of these sources makes the experiments more difficult to control and to reproduce [168]. Only a few works used a real infrastructure. [153] proposed GreenSlot, a scheduler aware of renewable energy production and prediction, which was partly evaluated with a 16-node cluster connected to a PV panel array. The same authors also proposed GreenSwitch [168] consisting of a modified version of Hadoop able to take advantage of renewable energy production. This approach was evaluated on a real infrastructure composed of 64 servers, batteries and PV panels. Moreover, in both simulations or small-scale experiments, a single electrical line is used to link the power sources, electrical storage and IT infrastructure (including cooling system, servers and networking devices). It is then, difficult to reproduce real conditions of datacenters.

\section{Acknowledgements}

This work was partly supported by the French Research Agency under the DataZero project (ANR-15-CE25-0012) and the EIPHI Graduate School (contract "ANR-17-EURE-0002").

\section{References}

[1] George Kamiya. Data centres and data transmission networks: Tracking report - june 2020. Technical report, Technical report, IEA, Northwestern University, 2020.

[2] Frédéric Bordage. Environmental footprint of the digital world. Technical report, GreenIT.fr, 2019.

[3] Hugues Ferreboeuf. Lean ICT: Towards digital sobriety. Technical report, The Shift Project, 2019.

[4] ITU-T. Greenhouse gas emissions trajectories for the information and communication technology sector compatible with the unfccc paris agreement. Technical Report ITU-T L.1470, International Telecommunication Union, 2020.

[5] Anne-Cécile Orgerie, Marcos Dias de Assunção, and Laurent Lefèvre. A survey on techniques for improving the energy efficiency of large scale distributed systems. ACM Computing Surveys, 46(4), April 2014.

[6] Anton Beloglazov, Rajkumar Buyya, Young Choon Lee, and Albert Y. Zomaya. A taxonomy and survey of energy-efficient data centers and cloud computing systems. Advances in Computers, 82:47-111, 2011. URL http://dblp.uni-trier.de/db/journals/ac/ac82 . html\#BeloglazovBLZ11.

[7] Toni Mastelic, Ariel Oleksiak, Holger Claussen, Ivona Brandic, Jean-Marc Pierson, and Athnasios V. Vasilakos. Cloud Computing: Survey on Energy Efficiency. ACM Computing Surveys, 47(2):1-36, December 2014. URL http://oatao.univ-toulouse.fr/13283/.

[8] Muhammad Zakarya and Lee Gillam. Energy efficient computing, clusters, grids and clouds: A taxonomy and survey. Sustainable Computing: Informatics and Systems, 14:13 - 33, 2017. doi: 10.1016/j.suscom.2017.03.002.

[9] Bijen R. Shrestha, Ujjwol Tamrakar, Timothy M. Hansen, Bishnu P. Bhattarai, Sean James, and Reinaldo Tonkoski. Efficiency and reliability analyses of AC and $380 \mathrm{v}$ DC distribution in data centers. IEEE Access, 6:63305-63315, 2018. doi: 10.1109/access.2018.2877354. URL https://doi.org/10.1109/access.2018.2877354.

[10] Khosrow Ebrahimi, Gerard F. Jones, and Amy S. Fleischer. A review of data center cooling technology, operating conditions and the corresponding low-grade waste heat recovery opportunities. Renewable and Sustainable Energy Reviews, 31:622 - 638, 2014. ISSN 1364-0321. doi: https://doi.org/10.1016/j.rser.2013.12.007. URL http://www.sciencedirect.com/science/article/pii/ S1364032113008216.

[11] Khosrow Ebrahimi, Gerard F Jones, and Amy S Fleischer. Thermo-economic analysis of steady state waste heat recovery in data centers using absorption refrigeration. Applied Energy, 139:384-397, 2015.

[12] Shihong Zeng, Yuchen Liu, Chao Liu, and Xin Nan. A review of renewable energy investment in the brics countries: History, models, problems and solutions. Renewable and Sustainable Energy Reviews, 74:860-872, 2017. ISSN 1364-0321. doi: https://doi.org/10.1016/j. rser.2017.03.016. URL https://www.sciencedirect.com/science/article/pii/S1364032117303325.

[13] Shihong Zeng, Jingmin Jia, Bin Su, Chunxia Jiang, and Guowang Zeng. The volatility spillover effect of the european union (eu) carbon financial market. Journal of Cleaner Production, 282:124394, 2021. ISSN 0959-6526. doi: https://doi.org/10.1016/j.jclepro.2020.124394. URL https://www.sciencedirect.com/science/article/pii/S0959652620344395.

[14] Apple. Environmental responsibility report. Technical report, Apple, 2019.

[15] Yevgeniy Sverdlik. Largest solar farms ever built for google to power its southeast data centers. datacenterknowledge, 2019.

[16] Green Data Net. Green data net project, 2016. http://www.greendatanet-project.eu/home.html. 
[17] Ali Hammadi and Lotfi Mhamdi. A survey on architectures and energy efficiency in data center networks. Computer Communications, 40: 1 - 21, 2014. ISSN 0140-3664. doi: 10.1016/j.comcom.2013.11.005.

[18] M Arlitt, C Bash, S Blagodurov, Y Chen, T Christian, D Gmach, C Hyser, N Kumari, Z Liu, M Marwah, et al. Towards the design and operation of net-zero energy data centers. In 13th IEEE Intersociety Conference on Thermal and Thermomechanical Phenomena in Electronic Systems (ITherm), pages 552-561, 2012.

[19] Eduard Oró, Victor Depoorter, Albert Garcia, and Jaume Salom. Energy efficiency and renewable energy integration in data centres. strategies and modelling review. Renewable and Sustainable Energy Reviews, 42:429-445, 2015.

[20] RenewIT. Renewit project, 2016. http://www.renewit-project.eu/.

[21] X. You, Y. Li, M. Zheng, C. Zhu, and L. Yu. A survey and taxonomy of energy efficiency relevant surveys in cloud-related environments. IEEE Access, 5:14066-14078, 2017. ISSN 2169-3536. doi: 10.1109/ACCESS.2017.2718001.

[22] Muhammad Zakarya. Energy, performance and cost efficient datacenters: A survey. Renewable and Sustainable Energy Reviews, 94:363 385, 2018. ISSN 1364-0321. doi: 10.1016/j.rser.2018.06.005.

[23] Fanxin Kong and Xue Liu. A survey on green-energy-aware power management for datacenters. ACM Comput. Surv., 47(2):30:1-30:38, November 2014. ISSN 0360-0300. doi: 10.1145/2642708.

[24] C. Ren, D. Wang, B. Urgaonkar, and A. Sivasubramaniam. Carbon-aware energy capacity planning for datacenters. In IEEE 20th International Symposium on MASCOTS'12, pages 391-400, Aug 2012.

[25] Economic Development Blog. MidAmerican Energy to Invest \$1.9B for Iowa Wind Generation, 2013.

[26] Urs Hölzle. 100\% renewable is just the beginning, 2016. https://sustainability.google/projects/announcement-100/.

[27] F. Yang and A. A. Chien. Large-scale and extreme-scale computing with stranded green power: Opportunities and costs. IEEE Transactions on Parallel and Distributed Systems, 29(5):1103-1116, May 2018.

[28] eBay Inc. New utah data center advances our commitment to greener commerce, 2013. https://www.ebayinc.com/stories/news/ new-utah-data-center-advances-our-commitment-greener-commerce/.

[29] datacenterknowledge. The apple data center faq, part 2, 2012. https://www.datacenterknowledge.com/data-center-faqs/ apple-data-center-faq-part-2/.

[30] Greenpeace Inc. Clicking clean: how companies are creating the green internet, 2014. Available: https://goo.gl/7XJsh5.

[31] Tessaleno Devezas, David LePoire, João CO Matias, and Abílio MP Silva. Energy scenarios: Toward a new energy paradigm. Futures, 40 (1):1-16, 2008.

[32] Khosrow Moslehi and Ranjit Kumar. A reliability perspective of the smart grid. IEEE Transactions on Smart Grid, 1(1):57-64, 2010.

[33] Deepak Paramashivan Kaundinya, P Balachandra, and NH Ravindranath. Grid-connected versus stand-alone energy systems for decentralized power-a review of literature. Renewable and Sustainable Energy Reviews, 13(8):2041-2050, 2009.

[34] Bianca van der Ha, Paul Stoelinga, Mieke Timmerman, Hans Trapman, Stephan Zilder, Noah Pflugradt, Francisco Diaz González, Eduard Oro, and Davide Cesarini Nardi. Renewit deliverable d7.2: Overview of potential solutions for data centers case studies. Technical report, RenewIt EU Project, 2015. URL http://www.renewit-project.eu/wp-content/files_mf/ 14449875370verviewof potentialsolutionsforDataCentrescasestudies . pdf.

[35] Mark Z. Jacobson, Mark A. Delucchi, Zack A.F. Bauer, Savannah C. Goodman, William E. Chapman, Mary A. Cameron, Cedric Bozonnat, Liat Chobadi, Hailey A. Clonts, Peter Enevoldsen, Jenny R. Erwin, Simone N. Fobi, Owen K. Goldstrom, Eleanor M. Hennessy, Jingyi Liu, Jonathan Lo, Clayton B. Meyer, Sean B. Morris, Kevin R. Moy, Patrick L. O’Neill, Ivalin Petkov, Stephanie Redfern, Robin Schucker, Michael A. Sontag, Jingfan Wang, Eric Weiner, and Alexander S. Yachanin. 100\% clean and renewable wind, water, and sunlight all-sector energy roadmaps for 139 countries of the world. Joule, 1(1):108 - 121, 2017. ISSN 2542-4351. doi: https://doi.org/10.1016/j.joule.2017. 07.005. URL http://www. sciencedirect.com/science/article/pii/S2542435117300120.

[36] Anya Castillo and Dennice F. Gayme. Grid-scale energy storage applications in renewable energy integration: A survey. Energy Conversion and Management, 87:885 - 894, 2014. ISSN 0196-8904. doi: https://doi.org/10.1016/j.enconman.2014.07.063. URL http: //www.sciencedirect.com/science/article/pii/s0196890414007018.

[37] Raquel S Garcia and Daniel Weisser. A wind-diesel system with hydrogen storage: Joint optimisation of design and dispatch. Renewable energy, 31(14):2296-2320, 2006.

[38] Weiqiang Dong, Yanjun Li, and Ji Xiang. Optimal sizing of a stand-alone hybrid power system based on battery/hydrogen with an improved ant colony optimization. Energies, 9(10):785, 2016.

[39] J Zeng, M Li, JF Liu, J Wu, and HW Ngan. Operational optimization of a stand-alone hybrid renewable energy generation system based on an improved genetic algorithm. In IEEE Power and Energy Society General Meeting, pages 1-6, 2010.

[40] HX Yang, L Lu, and J Burnett. Weather data and probability analysis of hybrid photovoltaic-wind power generation systems in hong kong. Renewable Energy, 28(11):1813-1824, 2003.

[41] G Tina and S Gagliano. Probabilistic analysis of weather data for a hybrid solar/wind energy system. International Journal of Energy Research, 35(3):221-232, 2011.

[42] Datacenter Forum. Amazon's first operational wind farm in europe delivers clean energy to sweden, 2020. https://www. datacenter-forum.com/datacenter-forum/amazons-first-operational-wind-farm-in-europe-delivers-clean-energy-to-swed

[43] Pete Danko. How oregon projects are helping apple reach its green goals, 2020. ttps://www.bizjournals.com/portland/news/ 2020/07/21/apples-oregon-renewable-energy-projects.html.

[44] Sebastian Moss. Windcores project deploys small data centers inside wind turbines, 2018. https://www.datacenterdynamics . com/ en/news/windcores-project-deploys-small-data-centers-inside-wind-turbines/.

[45] Gilbert M. Masters. Renewable and efficient electric power systems. John Wiley \& Sons, standford university edition, 2013.

[46] Marco Bortolini, Mauro Gamberi, and Alessandro Graziani. Technical and economic design of photovoltaic and battery energy storage system. Energy Conversion and Management, 86:81-92, 2014.

[47] Mohammad Masih Sediqi, Masahiro Furukakoi, Mohammed E Lotfy, Atsushi Yona, and Tomonobu Senjyu. Optimal economical sizing of 
grid-connected hybrid renewable energy system. Journal of Energy and Power Engineering, 11(4):244-53, 2017.

[48] JK Kaldellis, D Zafirakis, and E Kondili. Optimum autonomous stand-alone photovoltaic system design on the basis of energy pay-back analysis. Energy, 34(9):1187-1198, 2009.

[49] Sunanda Sinha and SS Chandel. Review of recent trends in optimization techniques for solar photovoltaic-wind based hybrid energy systems. Renewable and Sustainable Energy Reviews, 50:755-769, 2015.

[50] Christian Roselund. New mexico prepares to approve $100 \mathrm{mw}$-ac of solar for facebook data centers, 2018. https: //www . pv-magazine . com/2018/09/07/new-mexico-prepares-to-approve-100-mw-ac-of-solar-for-facebook-data-centers.

[51] Steve Ranger. Google: 1.6 million solar panels will power these new data centres, 2019. https://www.zdnet.com/article/ google-1-6-million-solar-panels-will-power-these-new-data-centres/.

[52] Emiliano Bellini. Another facebook data center goes solar, 2018. https://www.pv-magazine.com/2018/09/18/ another-facebook-data-center-goes-solar/.

[53] Nand Kishor, RP Saini, and SP Singh. A review on hydropower plant models and control. Renewable and Sustainable Energy Reviews, 11 (5):776-796, 2007.

[54] datacenterlight. 99.9\% hydropower: Not every datacenter has in-house hydropower plant. we do. data center light operates with $99.9 \mathrm{https}$ : //datacenterlight.ch/en-us/cms/hydropower/.

[55] Pete Danko. Facebook does hydro: Sweden data center opens, 2013. https://www.greentechmedia.com/articles/read/ facebook-does-hydro-sweden-data-center-opens.

[56] Robert McMillan. Apple buys tiny dams to power its data centers, 2014. https://www . wired.com/2014/04/ebd/.

[57] Water Power. Hydro power's volkswagen data center in norway, 2019. https://www.waterpowermagazine.com/news/ newshydro-powers-volkswagen-data-center-in-norway-7281947.

[58] Tryggvi Adalbjornsson. Iceland's data centers are booming-here's why that's a problem, 2019. https: //www . technologyreview . com/ 2019/06/18/134902/icelands-data-centers-are-booming-heres-why-thats-a-problem/.

[59] SR Tewfik. Biomass utilization facilities and biomass processing technologies. Energy Educ. Sc. and Tech., 14:1-19, 2004.

[60] RK Chakrabarty, H Moosmüller, L-WA Chen, K Lewis, WP Arnott, C Mazzoleni, MK Dubey, CE Wold, WM Hao, and SM Kreidenweis. Brown carbon in tar balls from smoldering biomass combustion. Atmospheric Chemistry and Physics, 10(13):6363-6370, 2010.

[61] Qi Zhang, Jie Chang, Tiejun Wang, and Ying Xu. Review of biomass pyrolysis oil properties and upgrading research. Energy conversion and management, 48(1):87-92, 2007.

[62] Takuya Yoshida, Yoshito Oshima, and Yukihiko Matsumura. Gasification of biomass model compounds and real biomass in supercritical water. Biomass and Bioenergy, 26(1):71-78, 2004.

[63] Saqib Sohail Toor, Lasse Rosendahl, and Andreas Rudolf. Hydrothermal liquefaction of biomass: a review of subcritical water technologies. Energy, 36(5):2328-2342, 2011.

[64] Miguel Herrero, Jose A Mendiola, Alejandro Cifuentes, and Elena Ibáñez. Supercritical fluid extraction: recent advances and applications. Journal of Chromatography a, 1217(16):2495-2511, 2010.

[65] JD Bryers. Structured modeling of the anaerobic digestion of biomass particulates. Biotechnology and Bioengineering, 27(5):638-649, 1985.

[66] Yan Lin and Shuzo Tanaka. Ethanol fermentation from biomass resources: current state and prospects. Applied Microbiology and Biotechnology, 69(6):627-642, 2006.

[67] Elisa Greene. Hp labs designs data center fueled by manure, 2010. https://www8.hp.com/us/en/hp-news/press-release.html? $i d=459491$.

[68] Jack Pouchet. Biomass-powered data centers: Next step for green it?, 2012. URL https://www . environmentalleader . com/2011/07/ biomass-powered-data-centers-next-step-for-green-it/.

[69] Nana Yaw Amponsah, Mads Troldborg, Bethany Kington, Inge Aalders, and Rupert Lloyd Hough. Greenhouse gas emissions from renewable energy sources: A review of lifecycle considerations. Renewable and Sustainable Energy Reviews, 39:461 - 475, 2014.

[70] LAZARD. Lazard's levelized cost of energy analysis - version 14.0, 10 2020. URL https://www.lazard.com/media/451419/ lazards-levelized-cost-of-energy-version-140.pdf.

[71] Zachariah Iverson, Ajit Achuthan, Pier Marzocca, and Daryush Aidun. Optimal design of hybrid renewable energy systems (HRES) using hydrogen storage technology for data center applications. Renewable Energy, 52:79-87, 2013.

[72] SR Vosen and JO Keller. Hybrid energy storage systems for stand-alone electric power systems: optimization of system performance and cost through control strategies. International Journal of Hydrogen Energy, 24(12):1139-1156, 1999.

[73] MT Iqbal. Simulation of a small wind fuel cell hybrid energy system. Renewable Energy, 28(4):511-522, 2003.

[74] LA Torres, FJ Rodriguez, and PJ Sebastian. Simulation of a solar-hydrogen-fuel cell system: results for different locations in Mexico. International Journal of Hydrogen Energy, 23(11):1005-1009, 1998.

[75] Th F El-Shatter, Mona N Eskandar, and Mohsen T El-Hagry. Hybrid PV/fuel cell system design and simulation. Renewable Energy, 27(3): 479-485, 2002.

[76] Marwa Haddad, Jean-Marc Nicod, and Marie-Cecile Marion-Pera. Hydrogen infrastructure: Data-center supply-refueling station synergy. In IEEE Vehicle Power and Propulsion Conference (VPPC'2017), pages 1-6, 12 2017. doi: 10.1109/VPPC.2017.8330978.

[77] Benjamin Storrow. How big batteries at data centers could replace power plants, 2018. https://www.scientificamerican.com/ article/how-big-batteries-at-data-centers-could-replace-power-plants/.

[78] Andy Colthorpe. Rwe's new data centre solution offers ups batteries dual use as grid-balancing asset, 2020. https://www. energy-storage.news/news/rwes-new-data-centre-solution-offers-ups-batteries-dual-use-as-grid-balanci.

[79] Adam Wierman, Zhenhua Liu, Iris Liu, and Hamed Mohsenian-Rad. Opportunities and challenges for data center demand response. In Green Computing Conference (IGCC), 2014 International, pages 1-10. IEEE, 2014.

[80] Baris Aksanli and Tajana Rosing. Providing regulation services and managing data center peak power budgets. In Design, Automation \& Test in Europe Conference \& Exhibition (DATE), 2014, pages 1-4. IEEE Conference Publications, 2014. doi: 10.7873/date.2014.156. URL 
A survey of challenges and solutions for the integration of renewable energy in datacenters

https://doi.org/10.7873/date.2014.156.

[81] Di Wang, Chuangang Ren, Anand Sivasubramaniam, Bhuvan Urgaonkar, and Hosam Fathy. Energy storage in datacenters: what, where, and how much? In Proceedings of the 12th ACM SIGMETRICS/PERFORMANCE joint international conference on Measurement and Modeling of Computer Systems, pages 187-198, 2012.

[82] Ilari Alaperä, Samuli Honkapuro, Ville Tikka, and Janne Paananen. Dual-purposing UPS batteries for energy storage functions: A business case analysis. Energy Procedia, 158:5061-5066, February 2019. doi: 10.1016/j.egypro.2019.01.645. URL https://doi .org/10.1016/ j.egypro.2019.01.645.

[83] Mary Branscombe. Google's solar deal for nevada data center would be largest of its kind, 2020. https: //www . datacenterknowledge . com/google-alphabet/google-s-solar-deal-nevada-data-center-would-be-largest-its-kind.

[84] J. Luo, L. Rao, and X. Liu. Spatio-temporal load balancing for energy cost optimization in distributed internet data centers. IEEE Transactions on Cloud Computing, 3(3):387-397, July 2015. ISSN 2168-7161. doi: 10.1109/TCC.2015.2415798.

[85] Murat Yilmaz and Philip T Krein. Review of battery charger topologies, charging power levels, and infrastructure for plug-in electric and hybrid vehicles. IEEE Transactions on Power Electronics, 28(5):2151-2169, 2013.

[86] Jeff Kessen. Why li-ion batteries are the progressive option for data centers, 2019. https://www.datacenterdynamics.com/en/ opinions/why-li-ion-batteries-are-progressive-option-data-centers/.

[87] George Kyriakarakos, Dimitrios D Piromalis, Konstantinos G Arvanitis, Anastasios I Dounis, and George Papadakis. On battery-less autonomous polygeneration microgrids: Investigation of the combined hybrid capacitors/hydrogen alternative. Energy Conversion and Management, 91:405-415, 2015.

[88] Giorgio Cau, Daniele Cocco, Mario Petrollese, Søren Knudsen Kær, and Christian Milan. Energy management strategy based on short-term generation scheduling for a renewable microgrid using a hydrogen storage system. Energy Conversion and Management, 87:820-831, 2014.

[89] O Erdinc and M Uzunoglu. A new perspective in optimum sizing of hybrid renewable energy systems: Consideration of component performance degradation issue. International Journal of Hydrogen Energy, 37(14):10479-10488, 2012.

[90] M Tanrioven and MS Alam. Reliability modeling and analysis of stand-alone PEM fuel cell power plants. Renewable Energy, 31(7):915-933, 2006.

[91] Binayak Bhandari, Kyung-Tae Lee, Gil-Yong Lee, Young-Man Cho, and Sung-Hoon Ahn. Optimization of hybrid renewable energy power systems: A review. International Journal of Precision Engineering and Manufacturing-Green Technology, 2(1):99-112, 2015.

[92] Bei Li, Robin Roche, and Abdellatif Miraoui. Microgrid sizing with combined evolutionary algorithm and MILP unit commitment. Applied Energy, 188:547-562, 2017.

[93] I Baniasad Askari and M Ameri. Optimal sizing of photovoltaic-battery power systems in a remote region in Kerman, Iran. Proceedings of the Institution of Mechanical Engineers, Part A: Journal of Power and Energy, 223(5):563-570, 2009.

[94] Mustafa Engin and Dilşad Engin. Sizing PV-wind hybrid energy system for lighting. International Journal of Development and Sustainability, 1(2):85-98, 2012

[95] Thomas Pregger, Daniela Graf, Wolfram Krewitt, Christian Sattler, Martin Roeb, and Stephan Möller. Prospects of solar thermal hydrogen production processes. International Journal of Hydrogen Energy, 34(10):4256-4267, 2009.

[96] John Roach. Microsoft tests hydrogen fuel cells for backup power at datacenters, 2020 . https://news.microsoft.com/ innovation-stories/hydrogen-datacenters/.

[97] Bei Li, Robin Roche, Damien Paire, and Abdellatif Miraoui. Sizing of a stand-alone microgrid considering electric power, cooling/heating, hydrogen loads and hydrogen storage degradation. Applied Energy, 205:1244-1259, 2017.

[98] O.C. Onar, M. Uzunoglu, and M.S. Alam. Dynamic modeling, design and simulation of a wind/fuel cell/ultra-capacitor-based hybrid power generation system. Journal of Power Sources, 161(1):707-722, October 2006. doi: 10.1016/j.jpowsour.2006.03.055. URL https: //doi . org/10.1016/j. jpowsour. 2006.03.055.

[99] L. Valverde, F. Rosa, A.J. del Real, A. Arce, and C. Bordons. Modeling, simulation and experimental set-up of a renewable hydrogen-based domestic microgrid. International Journal of Hydrogen Energy, 38(27):11672-11684, September 2013. doi: 10.1016/j.ijhydene.2013.06. 113. URL https://doi.org/10.1016/j.ijhydene.2013.06.113.

[100] LAZARD. Lazard's levelized cost of storage analysis - version 6.0, 10 2020. URL https://www.lazard.com/media/451418/ lazards-levelized-cost-of-storage-version-60.pdf.

[101] Berk Celik, Siddharth Suryanarayanan, Robin Roche, and Timothy M. Hansen. Quantifying the impact of solar photovoltaic and energy storage assets on the performance of a residential energy aggregator. IEEE Transactions on Sustainable Energy, 11(1):405-414, January 2020. doi: 10.1109/tste.2019.2892603. URL https://doi.org/10.1109/tste.2019.2892603.

[102] Robert Arno, Addam Friedl, Peter Gross, and Robert J. Schuerger. Reliability of data centers by tier classification. IEEE Transactions on Industry Applications, 48(2):777-783, March 2012. doi: 10.1109/tia.2011.2180872. URL https://doi.org/10.1109/tia.2011. 2180872.

[103] Neil Rasmussen. Determining total cost of ownership for data center and network room infrastructure. Relatório técnico, Schneider Electric, Paris, 8, 2011.

[104] G. Parise and L. Parise. Electrical distribution for a reliable data center. In 2012 IEEE Industry Applications Society Annual Meeting, pages 1-5, Oct 2012. doi: 10.1109/IAS.2012.6374107.

[105] S. Qi, W. Sun, and Y. Wu. Comparative analysis on different architectures of power supply system for data center and telecom center. In 2017 IEEE International Telecommunications Energy Conference (INTELEC), pages 26-29, Oct 2017.

[106] DATAZERO Consortium. Datazero D2.1 deliverable, 2015.

[107] Marwa Haddad, Georges Da Costa, Jean-Marc Nicod, Marie-Cécile Péra, Jean-Marc Pierson, Veronika Rehn-Sonigo, Patricia Stolf, and Christophe Varnier. Combined it and power supply infrastructure sizing for standalone green data centers. Sustainable Computing: Informatics and Systems, 30:100505, 2021.

[108] Ayham Kassab, Jean-Marc Nicod, Laurent Philippe, and Veronika Rehn-Sonigo. Green power aware approaches for scheduling independent 
tasks on a multi-core machine. Sustainable Computing: Informatics and Systems, 31:100590, 2021.

[109] M.A. Hannan, M. Faisal, Pin Jern Ker, R.A. Begum, Z.Y. Dong, and C. Zhang. Review of optimal methods and algorithms for sizing energy storage systems to achieve decarbonization in microgrid applications. Renewable and Sustainable Energy Reviews, 131:110022, 2020. ISSN 1364-0321. doi: https://doi.org/10.1016/j.rser.2020.110022. URL https://www.sciencedirect.com/science/article/pii/ S1364032120303130.

[110] Piyali Ganguly, Akhtar Kalam, and Aladin Zayegh. Solar-wind hybrid renewable energy system: current status of research on configurations, control, and sizing methodologies. In Hybrid-Renewable Energy Systems in Microgrids, pages 219-248. Elsevier, 2018. doi: 10.1016/ b978-0-08-102493-5.00012-1. URL https://doi .org/10.1016/b978-0-08-102493-5.00012-1.

[111] Yuqing Yang, Stephen Bremner, Chris Menictas, and Merlinde Kay. Battery energy storage system size determination in renewable energy systems: A review. Renewable and Sustainable Energy Reviews, 91:109-125, 2018. ISSN 1364-0321. doi: https://doi.org/10.1016/j.rser. 2018.03.047. URL https://www.sciencedirect.com/science/article/pii/S1364032118301436.

[112] Meiling Yue, Hugo Lambert, Elodie Pahon, Robin Roche, Samir Jemei, and Daniel Hissel. Hydrogen energy systems: A critical review of technologies, applications, trends and challenges. Renewable and Sustainable Energy Reviews, 146:111180, 2021. ISSN 1364-0321. doi: https://doi.org/10.1016/j.rser.2021.111180. URL https://www. sciencedirect.com/science/article/pii/S1364032121004688.

[113] C Protogeropoulos, BJ Brinkworth, and RH Marshall. Sizing and techno-economical optimization for hybrid solar photovoltaic/wind power systems with battery storage. International Journal of Energy Research, 21(6):465-479, 1997.

[114] Sunanda Sinha and SS Chandel. Review of software tools for hybrid renewable energy systems. Renewable and Sustainable Energy Reviews, 32:192-205, 2014.

[115] NREL. Hybrid optimization model for electric renewable energy, 2009. URL https://www . homerenergy . com/.

[116] Rodolfo Dufo-Lopez and José L Bernal-Agustín. Design and control strategies of PV-diesel systems using genetic algorithms. Solar Energy, 79(1):33-46, 2005.

[117] Hongxing Yang, Wei Zhou, Lin Lu, and Zhaohong Fang. Optimal sizing method for stand-alone hybrid solar-wind system with LPSP technology by using genetic algorithm. Solar Energy, 82(4):354-367, 2008.

[118] Hongxing Yang, Zhou Wei, and Lou Chengzhi. Optimal design and techno-economic analysis of a hybrid solar-wind power generation system. Applied Energy, 86(2):163-169, 2009.

[119] S Ashok. Optimised model for community-based hybrid energy system. Renewable Energy, 32(7):1155-1164, 2007.

[120] Singiresu S Rao. Engineering optimization: theory and practice. John Wiley \& Sons, 2009.

[121] Y. Li, A. C. Orgerie, and J. M. Menaud. Balancing the use of batteries and opportunistic scheduling policies for maximizing renewable energy consumption in a cloud data center. In 2017 25th Euromicro International Conference on Parallel, Distributed and Network-based Processing (PDP), pages 408-415, March 2017. doi: 10.1109/PDP.2017.24.

[122] José L Bernal-Agustín, Rodolfo Dufo-López, and David M Rivas-Ascaso. Design of isolated hybrid systems minimizing costs and pollutant emissions. Renewable Energy, 31(14):2227-2244, 2006.

[123] YA Katsigiannis, PS Georgilakis, and ES Karapidakis. Multiobjective genetic algorithm solution to the optimum economic and environmental performance problem of small autonomous hybrid power systems with renewables. IET Renewable Power Generation, 4(5):404-419, 2010.

[124] Jun-Hai Shi, Xin-Jian Zhu, and Guang-Yi Cao. Design and techno-economical optimization for stand-alone hybrid power systems with multi-objective evolutionary algorithms. International Journal of Energy Research, 31(3):315-328, 2007.

[125] Lingfeng Wang and Chanan Singh. Multicriteria design of hybrid power generation systems based on a modified particle swarm optimization algorithm. IEEE Transactions on Energy Conversion, 24(1):163-172, 2009.

[126] L Hontoria, J Aguilera, and P Zufiria. Generation of hourly irradiation synthetic series using the neural network multilayer perceptron. Solar Energy, 72(5):441-446, 2002.

[127] A Mellit, M Benghanem, A Hadj Arab, and A Guessoum. A simplified model for generating sequences of global solar radiation data for isolated sites: Using artificial neural network and a library of markov transition matrices approach. Solar Energy, 79(5):469-482, 2005.

[128] A Mellit, M Benghanem, A Hadj Arab, and A Guessoum. An adaptive artificial neural network model for sizing stand-alone photovoltaic systems: application for isolated sites in algeria. Renewable Energy, 30(10):1501-1524, 2005.

[129] L Hontoria, J Aguilera, and P Zufiria. A new approach for sizing stand alone photovoltaic systems based in neural networks. Solar Energy, 78(2):313-319, 2005.

[130] Adel Mellit, M Benghanem, and Soteris A Kalogirou. Modeling and simulation of a stand-alone photovoltaic system using an adaptive artificial neural network: Proposition for a new sizing procedure. Renewable energy, 32(2):285-313, 2007.

[131] Laura Siso, Jaume Salom, Eduard Oro, Georges Da Costa, and Thomas Zilio. CoolEmAll D5.6 Final metrics and benchmarks. Research report, IRIT-Institut de recherche en informatique de Toulouse, March 2014. URL https : / hal . archives-ouvertes .fr/hal- 01818070.

[132] Jaume Salom, Albert Garcia, Eduard Oró, and Davide Nardi Cesarini. Renewit deliverable d3.1 metrics for net zero energy data centres. Technical report, RenewIT EU Project, 2014. URL http://www.renewit-project.eu/wp-content/files_mf/1413980534D3. 1MetricsforNetZeroDataCentresv9.0_final.pdf.

[133] Milagros Rey Porto, Silvia Sanjoaquín Vives, Aleix Fonellosa Caro, Marta Chinnici, Andrea Quintiliani, Ariel Oleksiak, Georges Da Costa, Eduard Oró, Davide Nardi Cesarini, Philip Inglesant, Mario Macias, Jaume Salom, Vassilios Tsachouridis, Ton Engbersen, Tomas Fernandez Buckley, John Bynum, Vojta Zavrel, Robert Birke, Massey Elizabeth, Massimo Bertoncini, Arnone Diego, Terpsi Velivasaki, Ioan Salomie, Tudor Cioara, Fabrice Roudet, Nicolas Samman, and Artemis Voulkidis. Data Centres Sustainability Cluster Activities Task 3. Rapport de recherche 3, European Commission, octobre 2014. URL https://ec. europa. eu/digital-agenda/en/news/ cluster-fp7-projects-proposes-new-environmental-efficiency-metrics-data-centres.

[134] Alexis I Aravanis, Artemis Voulkidis, Jaume Salom, Jacinta Townley, Vasiliki Georgiadou, Ariel Oleksiak, Milagros Rey Porto, Fabrice Roudet, and Theodore Zahariadis. Metrics for assessing flexibility and sustainability of next generation data centers. In Globecom Workshops (GC Wkshps), 2015 IEEE, pages 1-6. IEEE, 2015.

[135] Minh-Thuyen Thi, Jean-Marc Pierson, and Georges Da Costa. Game-based negotiation between power demand and supply in green datacen- 
ters. In 2020 IEEE Intl Conf on Parallel \& Distributed Processing with Applications, Big Data \& Cloud Computing, Sustainable Computing \& Communications, Social Computing \& Networking (ISPA/BDCloud/SocialCom/SustainCom), pages 690-697. IEEE, 2020.

[136] Léo Grange, Georges Da Costa, and Patricia Stolf. Green IT scheduling for data center powered with renewable energy. Future Generation Computer Systems, 86:99-120, September 2018. doi: 10.1016/j.future.2018.03.049.

[137] Maroua Haddad, Jean-Marc Nicod, Marie-Cécile Péra, and Christophe Varnier. Stand-Alone Renewable Power System Scheduling for a Green Data-Center using Integer Linear Programming, Version 1. Research report, FEMTO-ST, March 2019. URL https://hal. archives-ouvertes.fr/hal-02081951.

[138] R. Palma-Behnke, C. Benavides, F. Lanas, B. Severino, L. Reyes, J. Llanos, and D. Sáez. A microgrid energy management system based on the rolling horizon strategy. IEEE Transactions on Smart Grid, 4(2):996-1006, 2013. doi: 10.1109/TSG.2012.2231440.

[139] Moslem Uddin, Mohd Fakhizan Romlie, Mohd Faris Abdullah, Syahirah Abd Halim, Ab Halim Abu Bakar, and Tan Chia Kwang. A review on peak load shaving strategies. Renewable and Sustainable Energy Reviews, 82:3323-3332, 2018. ISSN 1364-0321. doi: https: //doi.org/10.1016/j.rser.2017.10.056. URL https://www.sciencedirect.com/science/article/pii/S1364032117314272.

[140] Dipesh Lamsal, Victor Sreeram, Yateendra Mishra, and Deepak Kumar. Output power smoothing control approaches for wind and photovoltaic generation systems: A review. Renewable and Sustainable Energy Reviews, 113:109245, 2019. ISSN 1364-0321. doi: https://doi.org/10.1016/j.rser.2019.109245. URL https://www.sciencedirect.com/science/article/pii/S1364032119304459.

[141] Tarkeshwar Mahto and V. Mukherjee. Energy storage systems for mitigating the variability of isolated hybrid power system. Renewable and Sustainable Energy Reviews, 51:1564-1577, 2015. ISSN 1364-0321. doi: https://doi.org/10.1016/j.rser.2015.07.012. URL https: //www.sciencedirect.com/science/article/pii/S1364032115006590.

[142] Joseba Jimeno, Jon Anduaga, José Oyarzabal, and Asier Gil de Muro. Architecture of a microgrid energy management system. European Transactions on Electrical Power, 21(2):1142-1158, 2011. doi: https://doi.org/10.1002/etep.443. URL https : //onlinelibrary . wiley . com/doi/abs/10.1002/etep. 443.

[143] Muhammad Fahad Zia, Elhoussin Elbouchikhi, and Mohamed Benbouzid. Microgrids energy management systems: A critical review on methods, solutions, and prospects. Applied Energy, 222:1033 - 1055, 2018. ISSN 0306-2619. doi: https://doi.org/10.1016/j.apenergy.2018. 04.103. URL http://www.sciencedirect.com/science/article/pii/S0306261918306676.

[144] Wei Deng, Fangming Liu, Hai Jin, and Xiaofei Liao. Online control of datacenter power supply under uncertain demand and renewable energy. In Communications (ICC), 2013 IEEE International Conference on, pages 4228-4232. IEEE, 2013.

[145] Chao Li, Rui Wang, Tao Li, Depei Qian, and Jingling Yuan. Managing green datacenters powered by hybrid renewable energy systems. In The 11th International Conference on Autonomic Computing, pages 261-272. USENIX, 2014.

[146] Enida Sheme, Patricia Stolf, Georges Da Costa, Jean-Marc Pierson, and Neki Frashëri. Efficient energy sources scheduling in green powered datacenters: A cloudsim implementation. NESUS workshop, 2016.

[147] Zhenhua Liu, Yuan Chen, Cullen Bash, Adam Wierman, Daniel Gmach, Zhikui Wang, Manish Marwah, and Chris Hyser. Renewable and cooling aware workload management for sustainable data centers. SIGMETRICS Perform. Eval. Rev., 40(1):175-186, 2012.

[148] Giuseppe Leo Guizzi and Michele Manno. Fuel cell-based cogeneration system covering data centers' energy needs. Energy, 41(1):56-64, 2012.

[149] Longjun Liu, Chao Li, Hongbin Sun, Yang Hu, Juncheng Gu, Tao Li, Jingmin Xin, and Nanning Zheng. HEB: deploying and managing hybrid energy buffers for improving datacenter efficiency and economy. In ACM SIGARCH Comp. Arch. News, volume 43, pages 463-475, 2015.

[150] Jeffrey D. Ullman. Np-complete scheduling problems. Journal of Computer and System sciences, 10(3):384-393, 1975.

[151] Mahdi Ghamkhari and Hamed Mohsenian-Rad. Energy and performance management of green data centers: A profit maximization approach. IEEE Transactions on Smart Grid, 4(2):1017-1025, 2013.

[152] Xiaoying Wang, Zhihui Du, Yinong Chen, and Mengqin Yang. A green-aware virtual machine migration strategy for sustainable datacenter powered by renewable energy. Simulation Modelling Practice and Theory, 58:3-14, 2015.

[153] Inigo Goiri, Md E. Haque, Kien Le, Ryan Beauchea, Thu D. Nguyen, Jordi Guitart, Jordi Torres, and Ricardo Bianchini. Matching renewable energy supply and demand in green datacenters. Ad Hoc Networks, 25:520 - 534, 2015. doi: 10.1016/j.adhoc.2014.11.012.

[154] Sergio Nesmachnow, Cristian Perfumo, and Íñigo Goiri. Holistic multiobjective planning of datacenters powered by renewable energy. Cluster Computing, 18(4):1379-1397, Dec 2015. ISSN 1573-7543. doi: 10.1007/s10586-015-0485-1.

[155] Hongtao Lei, Tao Zhang, Yajie Liu, Yabing Zha, and Xiaomin Zhu. SGEESS: Smart green energy-efficient scheduling strategy with dynamic electricity price for data center. Journal of Systems and Software, 108:23 - 38, 2015. doi: 10.1016/j.jss.2015.06.026.

[156] Hongtao Lei, Rui Wang, Tao Zhang, Yajie Liu, and Yabing Zha. A multi-objective co-evolutionary algorithm for energy-efficient scheduling on a green data center. Computers \& Op. Research, 75:103-117, 2016.

[157] Inès De Courchelle, Tom Guérout, Georges Da Costa, Thierry Monteil, and Yann Labit. Green energy efficient scheduling management. Simulation Modelling Practice and Theory, 2018. ISSN 1569-190X. doi: 10.1016/j.simpat.2018.09.011.

[158] Santiago Iturriaga and Sergio Nesmachnow. Scheduling energy efficient data centers using renewable energy. Electronics, 5(4):71, 2016.

[159] Sonja Klingert, Florian Niedermeier, Corentin Dupont, Giovanni Giuliani, Thomas Schulze, and Hermann de Meer. Renewable energy-aware data centre operations for smart cities the dc4cities approach. In IEEE Smart Cities and Green ICT Systems, pages 1-9, 2015.

[160] A. Kassab, J. M. Nicod, L. Philippe, and V. Rehn-Sonigo. Scheduling independent tasks in parallel under power constraints. In 46th International Conference on Parallel Processing (ICPP), pages 543-552, Aug 2017. doi: 10.1109/ICPP.2017.63.

[161] Ayham Kassab, Jean-Marc Nicod, Laurent Philippe, and Veronika Rehn-Sonigo. Assessing the use of genetic algorithms to schedule independent tasks under power constraints. In IEEE, Int. Conf. on High Perf. Computing \& Simulation (HPCS), pages 252-259, 2018.

[162] Stéphane Caux, Paul Renaud-Goud, Gustavo Rostirolla, and Patricia Stolf. IT optimization for datacenters under renewable power constraint. In Euro-Par 2018: Parallel Processing - 24th International Conference on Parallel and Distributed Computing, Turin, Italy, August 27-31, 2018, Proceedings, pages 339-351, 2018. doi: 10.1007/978-3-319-96983-1_24.

[163] Corentin Dupont, Mehdi Sheikhalishahi, Federico M Facca, and Fabien Hermenier. An energy aware application controller for optimizing 
A survey of challenges and solutions for the integration of renewable energy in datacenters

renewable energy consumption in data centres. In Utility and Cloud Computing (UCC), 2015 IEEE/ACM 8th International Conference on, pages 195-204. IEEE, 2015.

[164] Nicolas Beldiceanu, Bárbara Dumas Feris, Philippe Gravey, Sabbir Hasan, Claude Jard, Thomas Ledoux, Yunbo Li, Didier Lime, Gilles Madi-Wamba, Jean-Marc Menaud, Pascal Morel, Michel Morvan, Marie-Laure Moulinard, Anne-Cécile Orgerie, Jean-Louis Pazat, Olivier Roux, and Ammar Sharaiha. Towards energy-proportional clouds partially powered by renewable energy. Computing, 99(1):3-22, 2017. ISSN 1436-5057. doi: 10.1007/s00607-016-0503-z.

[165] Zhenhua Liu, Iris Liu, Steven Low, and Adam Wierman. Pricing data center demand response. SIGMETRICS Perform. Eval. Rev., 42(1): 111-123, June 2014. ISSN 0163-5999. doi: 10.1145/2637364.2592004.

[166] Tudor Cioara, Ionut Anghel, Marcel Antal, Sebastian Crisan, and Ioan Salomie. Data center optimization methodology to maximize the usage of locally produced renewable energy. In Sustainable Internet and ICT for Sustainability (SustainIT), 2015, pages 1-8. IEEE, 2015.

[167] Debdeep Paul, Wen-De Zhong, and Sanjay K Bose. Demand response in data centers through energy-efficient scheduling and simple incentivization. IEEE Systems Journal, 11(2):613-624, 2017.

[168] Íñigo Goiri, William Katsak, Kien Le, Thu D Nguyen, and Ricardo Bianchini. Parasol and greenswitch: Managing datacenters powered by renewable energy. ACM SIGARCH Computer Architecture News, 41(1):51-64, 2013.

[169] I. Goiri, W. Katsak, K. Le, T. D. Nguyen, and R. Bianchini. Designing and managing data centers powered by renewable energy. IEEE Micro, 34(3):8-16, May-June 2014. ISSN 0272-1732. doi: 10.1109/MM.2014.6.

[170] JM. Pierson, G. Baudic, S. Caux, B. Celik, G. Da Costa, L. Grange, M. Haddad, J. Lecuivre, J. Nicod, L. Philippe, V. Rehn-Sonigo, R. Roche, G. Rostirolla, A. Sayah, P. Stolf, M. Thi, and C. Varnier. Datazero: Datacenter with zero emission and robust management using renewable energy. IEEE Access, July 2019. ISSN 2169-3536. doi: 10.1109/ACCESS.2019.2930368.

[171] Zhenhua Liu, Minghong Lin, Adam Wierman, Steven H. Low, and Lachlan L.H. Andrew. Geographical load balancing with renewables. SIGMETRICS Perform. Eval. Rev., 39-3:62-66, December 2011. ISSN 0163-5999. doi: 10.1145/2160803.2160862.

[172] M. Lin, Z. Liu, A. Wierman, and L. L. H. Andrew. Online algorithms for geographical load balancing. In 2012 International Green Computing Conference (IGCC), pages 1-10, June 2012. doi: 10.1109/IGCC.2012.6322266.

[173] H. Goudarzi and M. Pedram. Force-directed geographical load balancing and scheduling for batch jobs in distributed datacenters. In IEEE International Conference on Cluster Computing (CLUSTER), pages 1-8, Sep. 2013.

[174] J. L. Berral, I. Goiri, T. D. Nguyen, R. Gavaldá, J. Torres, and R. Bianchini. Building green cloud services at low cost. In IEEE 34th International Conference on Distributed Computing Systems, pages 449-460, June 2014. doi: 10.1109/ICDCS.2014.53.

[175] Liang Zhang, Tao Han, and Nirwan Ansari. Renewable energy-aware inter-datacenter virtual machine migration over elastic optical networks. In IEEE International Conference on Cloud Computing Technology and Science (CloudCom), pages 440-443, 2015.

[176] C. Gu, C. Liu, J. Zhang, H. Huang, and X. Jia. Green scheduling for cloud data centers using renewable resources. In IEEE Conf. on Computer Communications Workshops (INFOCOM WKSHPS), pages 354-359, April 2015.

[177] C. Gu, H. Huang, and X. Jia. Green scheduling for cloud data centers using esds to store renewable energy. In 2016 IEEE International Conference on Communications (ICC), pages 1-7, May 2016. doi: 10.1109/ICC.2016.7511449.

[178] Debdeep Paul, Wen-De Zhong, and Sanjay K. Bose. Energy efficiency aware load distribution and electricity cost volatility control for cloud service providers. Journal of Network and Computer Applications, 59:185-197, 2016.

[179] X. Deng, D. Wu, J. Shen, and J. He. Eco-aware online power management and load scheduling for green cloud datacenters. IEEE Systems Journal, 10(1):78-87, 2016. doi: 10.1109/JSYST.2014.2344028.

[180] Adel Nadjaran Toosi, Chenhao Qu, Marcos Dias de Assuncao, and Rajkumar Buyya. Renewable-aware geographical load balancing of web applications for sustainable data centers. Journal of Network and Computer Applications, 83:155 - 168, 2017.

[181] Khosravi Atefeh, Nadjaran Toosi Adel, and Buyya Rajkumar. Online virtual machine migration for renewable energy usage maximization in geographically distributed cloud datacenters. Concurrency and Computation: Practice and Experience, 29(18):e4125, 2017.

[182] Y. Li, A. C. Orgerie, I. Rodero, M. Parashar, and J. M. Menaud. Leveraging renewable energy in edge clouds for data stream analysis in iot. In 17th IEEE/ACM Int. Symp. on Cluster, Cloud and Grid Computing (CCGRID), pages 186-195, May 2017.

[183] G. S. Aujla, N. Kumar, S. Garg, K. Kaur, R. Rajan, and S. Garg. Renewable energy-based multi-indexed job classification and container management scheme for sustainability of cloud data centers. IEEE Transactions on Industrial Informatics, pages 1-1, 2018. ISSN 1551-3203. doi: 10.1109/TII.2018.2800693.

[184] A. Rahman, X. Liu, and F. Kong. A survey on geographic load balancing based data center power management in the smart grid environment. IEEE Communications Surveys Tutorials, 16(1):214-233, 2014.

[185] Ali Habibi Khalaj, Khalid Abdulla, and Saman K. Halgamuge. Towards the stand-alone operation of data centers with free cooling and optimally sized hybrid renewable power generation and energy storage. Renewable and Sustainable Energy Reviews, 93:451-472, October 2018. ISSN 1364-0321. doi: 10.1016/j.rser.2018.05.006.

[186] Yanwei Zhang, Yefu Wang, and Xiaorui Wang. Testore: Exploiting thermal and energy storage to cut the electricity bill for datacenter cooling. In Proceedings of the 8th International Conference on Network and Service Management, CNSM '12, page 19-27, Laxenburg, AUT, 2012. International Federation for Information Processing. ISBN 9781450322102.

[187] Maciej Lukawski, Jefferson W. Tester, Michal C. Moore, Pawel Krol, and C. Lindsay Anderson. Demand Response for Reducing Coincident Peak Loads in Data Centers. In HICSS, page 11, January 2019. doi: 10.24251/HICSS.2019.413. URL http://hdl . handle . net/10125/ 59776.

[188] Yuling Li, Xiaoying Wang, Peicong Luo, and Qingyi Pan. Thermal-Aware Hybrid Workload Management in a Green Datacenter towards Renewable Energy Utilization. Energies, 12(8): 1494, April 2019. ISSN 1996-1073. doi: 10.3390/en12081494.

[189] Maël Madon and Jean-Marc Pierson. Integrating pre-cooling of data center operated with renewable energies. In 2020 International Conferences on Internet of Things (iThings) and IEEE Green Computing and Communications (GreenCom) and IEEE Cyber, Physical and Social Computing (CPSCom) and IEEE Smart Data (SmartData) and IEEE Congress on Cybermatics (Cybermatics), pages 332-341, 2020. doi: 10.1109/iThings-GreenCom-CPSCom-SmartData-Cybermatics50389.2020.00068. 
[190] W. Deng, F. Liu, H. Jin, B. Li, and D. Li. Harnessing renewable energy in cloud datacenters: opportunities and challenges. IEEE Network, 28(1):48-55, January 2014. ISSN 0890-8044. doi: 10.1109/MNET.2014.6724106.

[191] S. M. Ali, M. Jawad, M. U. S. Khan, K. Bilal, J. Glower, S. C. Smith, S. U. Khan, K. Li, and A. Y. Zomaya. An ancillary services model for data centers and power systems. IEEE Transactions on Cloud Computing, pages 1-1, 2018.

[192] M. Ghasemi-Gol, Y. Wang, and M. Pedram. An optimization framework for data centers to minimize electric bill under day-ahead dynamic energy prices while providing regulation services. In Int. Green Comp. Conf., pages 1-9, Nov 2014.

[193] David Aikema, Rob Simmonds, and Hamidreza Zareipour. Delivering ancillary services with data centres. Sustainable Computing: Informatics and Systems, 3(3):172 - 182, 2013. ISSN 2210-5379. doi: 10.1016/j.suscom.2013.01.012. Selected papers from the 2012 IEEE International Green Computing Conference (IGCC 2012).

[194] Marcel Antal, Claudia Pop, Dan Valea, Tudor Cioara, Ionut Anghel, and Ioan Salomie. Optimizing data centres operation to provide ancillary services on-demand. In Jörn Altmann, Gheorghe Cosmin Silaghi, and Omer F. Rana, editors, Economics of Grids, Clouds, Systems, and Services, pages 133-146, Cham, 2016. Springer International Publishing. ISBN 978-3-319-43177-2.

[195] L. Cupelli, N. Barve, and A. Monti. Optimal sizing of data center battery energy storage system for provision of frequency containment reserve. In IECON 2017 - 43rd Annual Conference of the IEEE Industrial Electronics Society, pages 7185-7190, Oct 2017. doi: 10.1109/ IECON.2017.8217257.

[196] M. Ghamkhari and H. Mohsenian-Rad. Data centers to offer ancillary services. In 2012 IEEE Third International Conference on Smart Grid Communications (SmartGridComm), pages 436-441, Nov 2012. doi: 10.1109/SmartGridComm.2012.6486023.

[197] M. Ghamkhari, A. Wierman, and H. Mohsenian-Rad. Energy portfolio optimization of data centers. IEEE Transactions on Smart Grid, 8 (4):1898-1910, July 2017. ISSN 1949-3053. doi: 10.1109/TSG.2015.2510428.

[198] W. Zheng, K. Ma, and X. Wang. Hybrid energy storage with supercapacitor for cost-efficient data center power shaving and capping. IEEE Transactions on Parallel and Distributed Systems, 28(4):1105-1118, April 2017.

[199] energyvault. Wind power and energy storage converge in the name of circular innovation, 2021. https://energyvault.ch/.

[200] cctenergystorage. Climate change technologies, 2021. https://www. cctenergystorage.com/.

[201] J. F. Torres, A. Troncoso, I. Koprinska, Z. Wang, and F. Martínez-Álvarez. Deep learning for big data time series forecasting applied to solar power. In Int. Joint Conference SOCO'18-CISIS'18-ICEUTE'18, pages 123-133, Cham, 2019. Springer.

[202] H. Casanova. Simgrid: A toolkit for the simulation of application scheduling. In First IEEE/ACM International Symposium on Cluster Computing and the Grid, 2001. Proceedings, pages 430-437, 2001. doi: 10.1109/CCGRID.2001.923223.

[203] Rodrigo N. Calheiros, Rajiv Ranjan, Anton Beloglazov, César A. F. De Rose, and Rajkumar Buyya. CloudSim: A toolkit for modeling and simulation of cloud computing environments and evaluation of resource provisioning algorithms. Software: Practice and Experience, 41(1): 23-50, January 2011. ISSN 1097-024X. doi: 10.1002/spe.995.

[204] K. Kurowski, A. Oleksiak, W. Piatek, T. Piontek, A. Przybyszewski, and J. Weglarz. DCworms - A tool for simulation of energy efficiency in distributed computing infrastructures. Simulation Modelling Practice and Th., 39:135-151, December 2013.

[205] John Wilkes and Charles Reiss. Details of the clusterdata-2011-1 trace, 2011. https://code.google.com/p/googleclusterdata/ wiki/ClusterData20111.

[206] Charles Reiss, Alexey Tumanov, Gregory R Ganger, Randy H Katz, and Michael A Kozuch. Heterogeneity and dynamicity of clouds at scale: Google trace analysis. In Proceedings of the Third ACM Symposium on Cloud Computing, page 7. ACM, 2012.

[207] Mansaf Alam, Kashish Ara Shakil, and Shuchi Sethi. Analysis and clustering of workload in google cluster trace based on resource usage. In Computational Science and Engineering (CSE) and IEEE Intl Conference on Embedded and Ubiquitous Computing (EUC) and 15th Intl Symposium on Distributed Computing and Applications for Business Engineering (DCABES), 2016 IEEE Intl Conference on, pages $740-747$. IEEE, 2016.

[208] Georges Da Costa, Léo Grange, and Inès de Courchelle. Modeling, classifying and generating large-scale google-like workload. Sustainable Computing: Informatics and Systems, 2018.

[209] Alexandru Iosup, Hui Li, Mathieu Jan, Shanny Anoep, Catalin Dumitrescu, Lex Wolters, and Dick HJ Epema. The grid workloads archive. Future Generation Computer Systems, 24(7):672-686, 2008.

[210] Martin Arlitt and Tai Jin. A workload characterization study of the 1998 world cup web site. IEEE network, 14(3):30-37, 2000.

[211] Manajit Sengupta, Yu Xie, Anthony Lopez, Aron Habte, Galen Maclaurin, and James Shelby. The national solar radiation data base (NSRDB). Renewable and Sustainable Energy Reviews, 89:51-60, 2018.

[212] MG Bosilovich, R Lucchesi, and M Suarez. MERRA-2: File specification. https://gmao.gsfc.nasa.gov/pubs/docs/Bosilovich785.pdf, 2015.

[213] Lee Fingersh, Dave Simms, Maureen Hand, Dave Jager, Jason Cotrell, Mike Robinson, Scott Schreck, and Scott M Larwood. Wind tunnel testing of NREL's unsteady aerodynamics experiment. In 20th ASME Wind Energy Symposium, pages 1-7, 2001. doi: 10.2514/6.2001-35.

[214] Ines De Courchelle, Thierry Monteil, Yann Labit, and Tom Guerout. A data model for supplying a Data Center with several energy sources. In Proceedings of the Workshop on Smart and Sustainable City, pages 1033-1038. IEEE, 2016.

[215] Niangjun Chen, Xiaoqi Ren, Shaolei Ren, and Adam Wierman. Greening multi-tenant data center demand response. Performance Evaluation, 91:229-254, 2015. 